\title{
Helicity Precession of Spin-1/2 Particles in Weak Inertial and Gravitational Fields
}

\author{
Dinesh Singh ${ }^{a *}$ Nader Mobed ${ }^{a \dagger}$, Giorgio Papini ${ }^{a, b \ddagger}$ \\ ${ }^{a}$ Department of Physics, University of Regina, Regina, Saskatchewan, S4S 0A2, Canada \\ ${ }^{b}$ International Institute for Advanced Scientific Studies, 89019 Vietri sul Mare (SA), Italy
}

November 11, 2018

\begin{abstract}
We calculate the helicity and chirality effects experienced by a spin- $1 / 2$ particle subjected to classical electromagnetic and gravitational fields. The helicity evolution is then determined in the non-relativistic, relativistic, and ultra-relativistic regimes. We find that inertia-gravitation can distinguish between helicity and chirality. Helicity is not conserved, in general, even when the particles are massless. In this case, however, the inertial fields can hardly be applied to the fermions.
\end{abstract}

\section{Introduction}

Over the past forty years, experimental connections between inertia-gravitation and quantum mechanics have been established in a limited number of instances. They confirm that inertia and Newtonian gravitation affect particle wavefunctions in ways that are consistent with covariant generalizations of known wave equations. Typical examples are represented by the Schrödinger and Klein-Gordon equations that have been successfully used to describe the behaviour of superconducting electrons [1, 2] and neutrons [3] in inertial-gravitational fields in a quasi-classical regime. Though the lengths scales involved, of order $10^{-3} \mathrm{~cm}$ for superelectrons [4] and $10^{-13} \mathrm{~cm}$ for neutrons [5], are far from comparable to Planck's length, which is thought to mark the onset of quantum gravity, the results extend the validity of certain aspects of relativistic inertia-gravitation by about thirty orders of magnitude.

Quantum particles are sensitive probes of inertia and, ultimately, gravity []. The unavoidable presence of inertial effects in precise tests of fundamental theories requires an attentive study of all aspects of inertia. As the length scales decrease, fundamental quantum properties of particles, like spin and discrete symmetries, come into play [7. This is the case for spin-1/2 particles, where they can be used in a variety of experimental situations and energy ranges, while still retaining a non-classical behaviour.

Particle accelerators and storage rings are very apt tools for studying inertia, rotational inertia in particular. The forerunner of these studies is the work by Bell and Leinaas [8]. They were able to calculate the effect of the coupling of spin to the quantum contributions $\delta \omega$ to the angular velocity. This is a kind of quantum Mashhoon effect that is likely responsible for a residual electron depolarization in storage rings. Issues of interest concern the behaviour of helicity [9] and chirality in the presence of inertia. If $\omega$ is classical, then spin has a precession frequency that equals

\footnotetext{
*e-mail: singhd@uregina.ca

$\dagger$ e-mail: nader.mobed@uregina.ca

‡e-mail: papini@uregina.ca
} 
the orbital frequency for fermions with $g=2$. But when $\kappa \equiv(g-2) / 2 \neq 0$, the spin vector undergoes an additional precession of frequency $\kappa e B / m$ that is measured with extreme accuracy in muon $g-2$ experiments [10]. Persistent residual discrepancies between standard model calculations and experiment have then been interpreted by one of us [7] as possible violations of the discrete symmetries in rotational inertia. These would arise if the gyro-gravitational ratio of the muon differs from one. There is a similarity here with the electromagnetic case, where $g=2$ is required by the Dirac equation, but not by quantum electrodynamics. Some unresolved problems exist regarding the helicity of massless particles due to rotational inertia, which in Minkowski space-time is a conserved quantity. This property may not hold true in the presence of inertial-gravitational fields, as suggested by Mashhoon for photons [11, 12. Cai and Papini [13, 14] found that spin-rotation coupling induces oscillations between massive left-handed and right-handed neutrinos (with non-vanishing magnetic moment), and that these oscillations persist in the limit of vanishing mass. Mergulhão [15] concluded that the helicity of massless neutrinos is not conserved when gravitational fields are present. On the contrary, a calculation by Aldovrandi et alii [16] based on linearized, scalar quantum gravity shows that helicity is conserved for massless fermions.

Our purpose here is to re-examine some of these questions starting from Hamiltonians that can be derived directly from the covariant Dirac equation [17]. Within the context of general relativity, comprehensive studies of the Dirac equation were conducted by De Oliveira and Tiomno [18] and Peres [19. Other authors introduce inertial interactions by means of unitary transformations. This is the approach taken by Bell and Leinaas [8]. Their work represents an extension of well known properties of the Schrödinger equation to the relativistic regime, but cannot be readily applied to gravitational fields. More recently, Hehl and Ni 20] have derived a comprehensive Hamiltonian using special relativity, while Obukhov [21] has discussed some of the limits of the most frequently used approximations. Experimentally, the validity of the covariant Dirac equation in an inertial-gravitational context finds support in the tests of the Page-Werner 22 and Bonse-Wroblewski 23] effects, and in the fact that spin-rotation coupling faithfully reproduces the essential features of $g-2$ experiments without the introduction of ad hoc arguments [24, 25]. We use below the formalism of general relativity that treats both inertial and gravitational fields in a unified way, but also avail ourselves of solutions that are exact to first order in the weak field approximation [13 17. We then develop suitable low- and high-energy approximations.

The purpose of this paper is to study the time rate of change of helicity and chirality for a massive, accelerated, charged spin- $1 / 2$ particle with total magnetic moment $\mu=(1+\kappa) \mu_{0}$, where $\kappa \mu_{0}$ is the anomalous part of the magnetic moment of the particle and $\mu_{0}$ is the Bohr magneton. The paper is organized as follows. Section 2 describes the Dirac Hamiltonian for a spin-1/2 particle under non-uniform acceleration and rotation. In addition to the original Hamiltonian, low- and high-energy approximations corresponding to non-relativistic and ultra-relativistic particle motion are derived via the Foldy-Wouthuysen (FW) [26] and Cini-Touschek (CT) 27] transformations, respectively. In Section 3, we calculate the spin-flip transition rate for each of the representations of the Hamiltonian. This is followed in Section 4 by the evaluation of the helicity operator's time evolution. Here it is shown that a non-zero helicity precession emerges due solely to the gravitational interactions found in the Berry's phase approach [13, 28, 29, 30, even when the particle is massless. Section 5 describes the chiral transition rate for a spin-1/2 particle in accelerated motion, and is followed by the conclusions in Section 6 .

\section{Dirac Hamiltonian for an Accelerated Spin-1/2 Particle}

\section{$2.1 \quad$ Original Representation}

Given the covariant Dirac equation ${ }^{1}$,

$$
\left[i \gamma^{\mu}(x) D_{\mu}-\frac{m}{\hbar}\right] \psi(x)=0,
$$

\footnotetext{
${ }^{1}$ Geometrized units of $c=1$ are assumed throughout, where the metric has signature -2 . Space-time indices are denoted by Greek characters and range from 0 to 3 , while spatial indices use Latin characters and range from 1 to 3 .
} 
where $m$ is the particle rest mass, $D_{\mu} \equiv \nabla_{\mu}+i \Gamma_{\mu}$ is the covariant derivative operator with $\nabla_{\mu}$ the usual covariant derivative on index-labelled tensors, and $\Gamma_{\mu}$ is the spinor connection, we seek to derive a corresponding Dirac Hamiltonian in a general co-ordinate frame. The gamma matrices $\left\{\gamma^{\mu}(x)\right\}$ satisfy $\left\{\gamma^{\mu}(x), \gamma^{\nu}(x)\right\}=2 g^{\mu \nu}(x)$ and $D_{\mu} \gamma^{\nu}=0$. The metric is described as

$$
\boldsymbol{g}=\eta_{\hat{\mu} \hat{\nu}} \boldsymbol{e}^{\hat{\mu}} \otimes \boldsymbol{e}^{\hat{\nu}}
$$

where we use a set of orthonormal tetrads 31] $\left\{\boldsymbol{e}_{\hat{\mu}}\right\}$ and basis one-forms $\left\{\boldsymbol{e}^{\hat{\mu}}\right\}$ labelled by indices with carets and satisfying the condition $\left\langle\boldsymbol{e}^{\hat{\mu}}, \boldsymbol{e}_{\hat{\nu}}\right\rangle=\delta_{{ }_{\hat{\nu}}}$ to define a local Lorentz frame. With vierbein sets $\left\{e^{\hat{\alpha}}{ }_{\mu}\right\},\left\{e^{\mu}{ }_{\hat{\alpha}}\right\}$ satisfying $\boldsymbol{e}^{\hat{\alpha}}=e^{\hat{\alpha}}{ }_{\beta} \boldsymbol{e}^{\beta}$ and $\boldsymbol{e}_{\hat{\alpha}}=e_{\hat{\alpha}}^{\beta} \boldsymbol{e}_{\beta}$, such that

$$
\begin{aligned}
e^{\hat{\alpha}}{ }_{\mu} e_{\hat{\beta}}^{\mu} & =\delta_{\hat{\beta}}^{\hat{\alpha}} \\
e^{\mu}{ }_{\hat{\alpha}} e^{\hat{\alpha}} & =\delta_{\nu}^{\mu}{ }_{\nu} \\
g_{\mu \nu} & =\eta_{\hat{\alpha} \hat{\beta}} e^{\hat{\alpha}} e_{\nu} e_{\nu}^{\hat{\beta}},
\end{aligned}
$$

we can relate the general metric to its Minkowski counterpart. The spinor connection is then

$$
\Gamma_{\mu}=-\frac{1}{4} \sigma^{\alpha \beta}(x) \Gamma_{\alpha \beta \mu}=-\frac{1}{4} \sigma^{\hat{\alpha} \hat{\beta}} \Gamma_{\hat{\alpha} \hat{\beta} \hat{\mu}} e_{\mu}^{\hat{\mu}},
$$

where $\sigma^{\hat{\alpha} \hat{\beta}}=\frac{i}{2}\left[\gamma^{\hat{\alpha}}, \gamma^{\hat{\beta}}\right]$ are the Minkowski space-time spin matrices and from the Cartan equation of differential forms

$$
\mathrm{d} e^{\hat{\mu}}+\Gamma_{\hat{\beta} \hat{\alpha}}^{\hat{\mu}} e^{\hat{\alpha}} \wedge e^{\hat{\beta}}=0
$$

we obtain $\Gamma_{\hat{\alpha} \hat{\beta} \hat{\mu}}$, the Ricci rotation coefficients. It is shown that, by arranging (2.1) into a Schrödinger form, the Hamiltonian in general space-time co-ordinates is $i \hbar \partial_{0} \psi(x)=H \psi(x)$, where

$$
H=\left(g^{00}\right)^{-1} e_{\hat{\mu}}^{0}\left[\gamma^{\hat{\mu}} m+e_{\hat{\nu}}^{j}\left(\eta^{\hat{\mu} \hat{\nu}}-i \sigma^{\hat{\mu} \hat{\nu}}\right)\left(-i \hbar \nabla_{j}+\hbar \Gamma_{j}\right)\right]+\hbar \Gamma_{0} .
$$

The orthonormal tetrad [20] for a spin-1/2 particle under accelerated motion with spatial rotational freedom is

$$
\begin{aligned}
& \boldsymbol{e}_{\hat{0}}=(1+\boldsymbol{a} \cdot \boldsymbol{x})^{-1}\left[\partial_{0}-(\boldsymbol{\omega} \times \boldsymbol{x})^{k} \partial_{k}\right] \\
& \boldsymbol{e}_{\hat{k}}=\partial_{k}
\end{aligned}
$$

and the corresponding basis one-form is

$$
\begin{aligned}
\boldsymbol{e}^{\hat{0}} & =(1+\boldsymbol{a} \cdot \boldsymbol{x}) \mathrm{d} x^{0} \\
\boldsymbol{e}^{\hat{k}} & =\mathrm{d} x^{k}+(\boldsymbol{\omega} \times \boldsymbol{x})^{k} \mathrm{~d} x^{0},
\end{aligned}
$$

where the three-acceleration $\boldsymbol{a}$ of the particle's frame and the rotation $\boldsymbol{\omega}$ of its spatial triad are generated by external electromagnetic fields. By introducing the electromagnetic potential and weak gravitational potential via the covariant Berry's phase $\Phi_{\mathrm{G}}$ [17, it follows from (2.7) that the Hamiltonian generated from (2.8) and (2.9) is

$$
\begin{aligned}
H= & (1+\boldsymbol{a} \cdot \boldsymbol{x})\left[\boldsymbol{\alpha} \cdot \boldsymbol{\pi}+m \beta+\frac{\kappa e \hbar}{2 m} \beta(i \boldsymbol{\alpha} \cdot \boldsymbol{E}-\boldsymbol{\sigma} \cdot \boldsymbol{B})\right]-\frac{i \hbar}{2}(\boldsymbol{\alpha} \cdot \boldsymbol{a}) \\
& -\boldsymbol{\omega} \cdot(\boldsymbol{x} \times \boldsymbol{\pi})-\frac{\hbar}{2} \boldsymbol{\sigma} \cdot \boldsymbol{\omega}+e \varphi+\boldsymbol{\alpha} \cdot\left(\boldsymbol{\nabla} \Phi_{\mathrm{G}}\right)+\left(\nabla_{0} \Phi_{\mathrm{G}}\right),
\end{aligned}
$$


where $\boldsymbol{\pi} \equiv \boldsymbol{p}-e \boldsymbol{A}$ with momentum operator $\boldsymbol{p}$ and electromagnetic vector potential $\boldsymbol{A}$, the anomalous magnetic moment $\kappa$ is inserted by hand, and the covariant Berry's phase is

$$
\Phi_{\mathrm{G}}=-\frac{1}{2} \int_{P}^{x} d z^{\lambda} \gamma_{\alpha \lambda}(z) p^{\alpha}+\frac{1}{2} \int_{P}^{x} d z^{\lambda}\left(\gamma_{\alpha \lambda, \beta}(z)-\gamma_{\beta \lambda, \alpha}(z)\right)\left(x^{\alpha}-z^{\alpha}\right) p^{\beta},
$$

where $p^{\mu}$ is the momentum eigenvalue of the free particle.

\subsection{Low- and High-Energy Approximations of the Hamiltonian}

It is possible to consider the precession of a spin- $1 / 2$ particle's helicity state for non-relativistic and ultra-relativistic motion. To do this, it is necessary that the Hamiltonian (2.10) undergo a suitable transformation which appropriately describes these energy limits. This is accomplished by using the Foldy-Wouthuysen (FW) [26, 32, and Cini-Touschek (CT) [27, 33] transformations to respectively obtain the low- and high-energy approximations of the Dirac Hamiltonian. For the special case of the free-particle Hamiltonian $H_{0}=m \beta+\boldsymbol{\alpha} \cdot \boldsymbol{\pi}$, the resulting low- and high-energy Hamiltonians $H_{0}^{\mathrm{FW}}$ and $H_{0}^{\mathrm{CT}}$ are well known. However, these derivations assume use of a Cartesian co-ordinate frame in performing the calculations. We want to generalize this approach by assuming a general curvilinear co-ordinate frame such that the results can then be applied to any orthogonal co-ordinate system. To accomplish this, we begin with the unitary operator $\exp \left(i S_{\mathrm{FW} / \mathrm{CT}}\right)$, where

$$
S_{\mathrm{FW} / \mathrm{CT}}=\frac{i}{2} \omega(q) \beta \frac{(\boldsymbol{\alpha} \cdot \boldsymbol{\pi})}{|\boldsymbol{\pi}|},
$$

the momentum operator $\boldsymbol{p}$ in curvilinear co-ordinates is

$$
P^{\hat{\imath}}=-i \hbar \nabla_{\hat{\imath}}=-i \hbar \frac{1}{\lambda^{\hat{\imath}}(u)} \frac{\partial}{\partial u^{\hat{\imath}}},
$$

with scale functions $\lambda^{\hat{\imath}}(u)$, and $\omega(q)$ is a constraint function dependent on $q \equiv m /|\boldsymbol{\pi}|$ and to be determined. ${ }^{2}$ Then

$$
H_{0}^{\mathrm{FW} / \mathrm{CT}}=e^{i S_{\mathrm{FW} / \mathrm{CT}}}[m \beta+\boldsymbol{\alpha} \cdot \boldsymbol{\pi}] e^{-i S_{\mathrm{FW} / \mathrm{CT}}}=e^{2 i S_{\mathrm{FW} / \mathrm{CT}}}[m \beta+\boldsymbol{\alpha} \cdot \boldsymbol{\pi}] .
$$

By Taylor expansion, it is shown that

$$
\begin{aligned}
e^{2 i S_{\mathrm{FW} / \mathrm{CT}}} & =1-\omega \beta \frac{(\boldsymbol{\alpha} \cdot \boldsymbol{\pi})}{|\boldsymbol{\pi}|}+\frac{1}{2 !}\left[\omega \beta \frac{(\boldsymbol{\alpha} \cdot \boldsymbol{\pi})}{|\boldsymbol{\pi}|}\right]^{2}-\frac{1}{3 !}\left[\omega \beta \frac{(\boldsymbol{\alpha} \cdot \boldsymbol{\pi})}{|\boldsymbol{\pi}|}\right]^{3}+\cdots \\
{\left[\omega \beta \frac{(\boldsymbol{\alpha} \cdot \boldsymbol{\pi})}{|\boldsymbol{\pi}|}\right]^{2} } & =-\frac{\omega^{2}}{|\boldsymbol{\pi}|^{2}}(\boldsymbol{\alpha} \cdot \boldsymbol{\pi})^{2}=-\frac{\omega^{2}}{|\boldsymbol{\pi}|^{2}}\left[\boldsymbol{\pi} \cdot \boldsymbol{\pi}+\frac{i}{2} \epsilon_{i j k} \sigma^{\hat{\imath}}\left[P^{\hat{\jmath}}, P^{\hat{k}}\right]-e \hbar \epsilon_{j k}^{i} \sigma^{\hat{k}}\left(\nabla_{\hat{\imath}} A^{\hat{\jmath}}\right)\right],
\end{aligned}
$$

It is an important point to recognize that, for general curvilinear co-ordinates, $\frac{i}{\hbar}\left[P^{\hat{\jmath}}, P^{\hat{k}}\right] \equiv N^{\hat{\jmath} \hat{k}} \neq 0$. Therefore, it follows that we can identify a vector operator $\boldsymbol{R}$ with the form

$$
\begin{aligned}
R^{\hat{k}}= & \frac{i}{2 \hbar} \epsilon_{i j}{ }^{k}\left[P^{\hat{\imath}}, P^{\hat{\jmath}}\right]=\frac{1}{2} \epsilon_{i j}{ }^{k} N^{\hat{\imath} \hat{\jmath}} \\
= & \delta^{k}{ }_{1}\left[\frac{1}{\lambda^{\hat{3}}(u)}\left(\frac{\partial}{\partial u^{\hat{3}}} \ln \lambda^{\hat{2}}(u)\right) P^{\hat{2}}-\frac{1}{\lambda^{\hat{2}}(u)}\left(\frac{\partial}{\partial u^{\hat{2}}} \ln \lambda^{\hat{3}}(u)\right) P^{\hat{3}}\right] \\
& +\delta^{k}{ }_{2}\left[\frac{1}{\lambda^{\hat{1}}(u)}\left(\frac{\partial}{\partial u^{\hat{1}}} \ln \lambda^{\hat{3}}(u)\right) P^{\hat{3}}-\frac{1}{\lambda^{\hat{3}}(u)}\left(\frac{\partial}{\partial u^{\hat{3}}} \ln \lambda^{\hat{1}}(u)\right) P^{\hat{1}}\right] \\
& +\delta^{k}{ }_{3}\left[\frac{1}{\lambda^{\hat{2}}(u)}\left(\frac{\partial}{\partial u^{\hat{2}}} \ln \lambda^{\hat{1}}(u)\right) P^{\hat{1}}-\frac{1}{\lambda^{\hat{1}}(u)}\left(\frac{\partial}{\partial u^{\hat{1}}} \ln \lambda^{\hat{2}}(u)\right) P^{\hat{2}}\right] .
\end{aligned}
$$

\footnotetext{
${ }^{2}$ We adopt the sign conventions used by Itzykson and Zuber [34 for the momentum operator and the three-dimensional Levi-Civita symbol, where $\epsilon^{i j k}=\epsilon_{i j k}$ and $\epsilon^{123} \equiv+1$, noting that the indices are raised and lowered with the Kronecker delta $\delta_{i j}$.
} 
Then, from (2.16), it is shown that

$$
\begin{aligned}
{\left[\omega \beta \frac{(\boldsymbol{\alpha} \cdot \boldsymbol{\pi})}{|\boldsymbol{\pi}|}\right]^{2} } & =-\omega^{2}\left[1+\frac{\hbar}{|\boldsymbol{\pi}|^{2}} \boldsymbol{\sigma} \cdot \boldsymbol{R}-\frac{e \hbar}{|\boldsymbol{\pi}|^{2}} \sigma^{\hat{k}} \epsilon_{j k}^{i}\left(\nabla_{\hat{\imath}} A^{\hat{\jmath}}\right)\right] \equiv-\chi^{2} \\
\chi & \approx \omega\left[1+\frac{\hbar}{2|\boldsymbol{\pi}|^{2}} \boldsymbol{\sigma} \cdot \boldsymbol{R}-\frac{e \hbar}{2|\boldsymbol{\pi}|^{2}} \sigma^{\hat{k}} \epsilon_{j k}^{i}\left(\nabla_{\hat{\imath}} A^{\hat{\jmath}}\right)\right] .
\end{aligned}
$$

Given (2.18), it is evident that $\boldsymbol{\sigma} \cdot \boldsymbol{R}$ resembles something like a magnetic dipole term due to the curl of the electromagnetic vector potential. However, this is interpreted as a purely co-ordinate-dependent effect due to the choice of momentum states defined in a particular co-ordinate system. For Cartesian co-ordinates, (2.17) identically vanishes. Therefore, by substituting into (2.15), we show that

$$
\begin{aligned}
e^{2 i S_{\mathrm{FW} / \mathrm{CT}}} & \approx \cos \chi-\sin \chi\left[\chi^{-1} \omega \beta \frac{(\boldsymbol{\alpha} \cdot \boldsymbol{\pi})}{|\boldsymbol{\pi}|}\right] \\
& \approx \cos \chi-\sin \chi\left[1-\frac{\hbar}{2|\boldsymbol{\pi}|^{2}} \boldsymbol{\sigma} \cdot \boldsymbol{R}+\frac{e \hbar}{2|\boldsymbol{\pi}|^{2}} \sigma^{\hat{k}} \epsilon_{j k}^{i}\left(\nabla_{\hat{\imath}} A^{\hat{\jmath}}\right)\right] \beta \frac{(\boldsymbol{\alpha} \cdot \boldsymbol{\pi})}{|\boldsymbol{\pi}|}
\end{aligned}
$$

and

$$
\begin{aligned}
H_{0}^{\mathrm{FW} / \mathrm{CT}} \approx & {\left[\cos \chi+q \sin \chi\left[1-\frac{\hbar}{2|\boldsymbol{\pi}|^{2}} \boldsymbol{\sigma} \cdot \boldsymbol{R}+\frac{e \hbar}{2|\boldsymbol{\pi}|^{2}} \sigma^{\hat{k}} \epsilon^{i}{ }_{j k}\left(\nabla_{\hat{\imath}} A^{\hat{\jmath}}\right)\right]\right](\boldsymbol{\alpha} \cdot \boldsymbol{\pi}) } \\
& +|\boldsymbol{\pi}|\left[q \cos \chi-\sin \chi\left[1+\frac{\hbar}{2|\boldsymbol{\pi}|^{2}} \boldsymbol{\sigma} \cdot \boldsymbol{R}-\frac{e \hbar}{2|\boldsymbol{\pi}|^{2}} \sigma^{\hat{k}} \epsilon_{j k}^{i}\left(\nabla_{\hat{\imath}} A^{\hat{\jmath}}\right)\right]\right] \beta .
\end{aligned}
$$

By setting the first coefficient of (2.20) to zero, we obtain the low-energy Hamiltonian, $H_{0}^{\mathrm{FW}}$, which amounts to solving for $\omega(q)$. It is straightforward to show that

$$
\begin{aligned}
\omega(q) & \approx\left[1-\frac{\hbar}{2|\boldsymbol{\pi}|^{2}} \boldsymbol{\sigma} \cdot \boldsymbol{R}+\frac{e \hbar}{2|\boldsymbol{\pi}|^{2}} \sigma^{\hat{k}} \epsilon_{j k}^{i}\left(\nabla_{\hat{\imath}} A^{\hat{\jmath}}\right)\right] \tan ^{-1}\left[-\frac{1}{q}\left[1+\frac{\hbar}{2|\boldsymbol{\pi}|^{2}} \boldsymbol{\sigma} \cdot \boldsymbol{R}-\frac{e \hbar}{2|\boldsymbol{\pi}|^{2}} \sigma^{\hat{k}} \epsilon_{j k}^{i}\left(\nabla_{\hat{\imath}} A^{\hat{\jmath}}\right)\right]\right] \\
& \approx-\frac{1}{q}=-\frac{|\boldsymbol{\pi}|}{m} \ll 1,
\end{aligned}
$$

with the result that

$$
H_{0}^{\mathrm{FW}}=\left[m+\frac{1}{2 m} \boldsymbol{\pi} \cdot \boldsymbol{\pi}+\frac{\hbar}{2 m} \boldsymbol{\sigma} \cdot \boldsymbol{R}-\frac{e \hbar}{2 m} \sigma^{\hat{k}} \epsilon_{j k}^{i}\left(\nabla_{\hat{\imath}} A^{\hat{\jmath}}\right)\right] \beta .
$$

In a local Cartesian frame, (2.22) becomes

$$
H_{0}^{\mathrm{FW}}=\left[m+\frac{1}{2 m} \boldsymbol{\pi} \cdot \boldsymbol{\pi}-\frac{e \hbar}{2 m} \boldsymbol{\sigma} \cdot \boldsymbol{B}\right] \beta
$$

where the last term is the familiar magnetic dipole moment. ${ }^{3}$

Similarly, setting the second coefficient of (2.20) to zero leads to the high-energy approximation, $H_{0}^{\mathrm{CT}}$. Following the same procedure, we show that

$$
\begin{aligned}
\omega(q) & \approx\left[1-\frac{\hbar}{2|\boldsymbol{\pi}|^{2}} \boldsymbol{\sigma} \cdot \boldsymbol{R}+\frac{e \hbar}{2|\boldsymbol{\pi}|^{2}} \sigma^{\hat{k}} \epsilon_{j k}^{i}\left(\nabla_{\hat{\imath}} A^{\hat{\jmath}}\right)\right] \tan ^{-1}\left[q\left[1-\frac{\hbar}{2|\boldsymbol{\pi}|^{2}} \boldsymbol{\sigma} \cdot \boldsymbol{R}+\frac{e \hbar}{2|\boldsymbol{\pi}|^{2}} \sigma^{\hat{k}} \epsilon_{j k}^{i}\left(\nabla_{\hat{\imath}} A^{\hat{\jmath}}\right)\right]\right] \\
& \approx q\left[1-\frac{\hbar}{|\boldsymbol{\pi}|^{2}} \boldsymbol{\sigma} \cdot \boldsymbol{R}+\frac{e \hbar}{|\boldsymbol{\pi}|^{2}} \sigma^{\hat{k}} \epsilon_{j k}^{i}\left(\nabla_{\hat{\imath}} A^{\hat{\jmath}}\right)\right] \ll 1,
\end{aligned}
$$

\footnotetext{
${ }^{3}$ We must note that $\epsilon_{j k}^{i}\left(\nabla_{\hat{\imath}} A^{\hat{\jmath}}\right)$ is not the kth component of the curl of $\boldsymbol{A}$ in curvilinear co-ordinates [35]. As well, all summations involving gradients throughout this paper, as defined by (2.13), are interpreted such that, for example, $\nabla_{\hat{k}} \nabla^{\hat{k}} \varphi=-\left[\nabla_{\hat{1}} \nabla_{\hat{1}}+\nabla_{\hat{2}} \nabla_{\hat{2}}+\nabla_{\hat{3}} \nabla_{\hat{3}}\right] \varphi$.
} 
and

$$
H_{0}^{\mathrm{CT}} \approx\left[\sqrt{|\boldsymbol{\pi}|^{2}+m^{2}}-\frac{q^{3}}{\sqrt{1+q^{2}}} \frac{\hbar}{2 m}\left[\boldsymbol{\sigma} \cdot \boldsymbol{R}-e \sigma^{\hat{k}} \epsilon^{i}{ }_{j k}\left(\nabla_{\hat{\imath}} A^{\hat{\jmath}}\right)\right]\right] \frac{(\boldsymbol{\alpha} \cdot \boldsymbol{\pi})}{|\boldsymbol{\pi}|} .
$$

Both (2.22) and (2.25) show that a small energy shift due to $\boldsymbol{R}$ emerges from applying the FW and CT transformations to $H_{0}$.

Having now obtained the means to extend the FW and CT transformations for a general curvilinear co-ordinate frame, we proceed to derive the low- and high-energy approximations of the Dirac Hamiltonian (2.10) for a non-uniformly accelerated spin- $1 / 2$ particle. Retaining all Hermitian terms up to order $1 / \mathrm{m}^{2}$, it is therefore shown that ${ }^{4}$

$$
\begin{aligned}
H_{\mathrm{FW}} \approx & (1+\boldsymbol{a} \cdot \boldsymbol{x})\left[m+\frac{1}{2 m} \boldsymbol{\pi} \cdot \boldsymbol{\pi}+\frac{\hbar}{2 m} \boldsymbol{\sigma} \cdot \boldsymbol{R}-\frac{e \hbar}{2 m} \sigma^{k} \epsilon_{j k}^{i}\left(\nabla_{i} A^{j}\right)\right] \beta-\frac{\kappa e \hbar}{2 m}(\boldsymbol{\sigma} \cdot \boldsymbol{B}) \beta \\
& +\frac{\hbar}{4 m}\left[\boldsymbol{\sigma} \cdot(\boldsymbol{\nabla}(\boldsymbol{a} \cdot \boldsymbol{x}) \times \boldsymbol{\pi})-\hbar\left(\nabla_{k} a^{k}\right)+\frac{\hbar}{2} \boldsymbol{\nabla} \cdot \boldsymbol{\nabla}(\boldsymbol{a} \cdot \boldsymbol{x})\right] \beta+\left[\frac{1}{m}\left(\boldsymbol{\nabla} \Phi_{\mathrm{G}}\right) \cdot \boldsymbol{\pi}+\frac{\hbar}{2 m} \sigma^{k} \epsilon^{i j}{ }_{k}\left(\nabla_{i} \nabla_{j} \Phi_{\mathrm{G}}\right)\right] \beta \\
& +\frac{e \hbar^{2}}{8 m^{2}}\left[\boldsymbol{\nabla} \cdot \boldsymbol{\nabla} \varphi-2 \kappa\left(\nabla_{k} E^{k}\right)\right]+\frac{e \hbar}{4 m^{2}} \boldsymbol{\sigma} \cdot[(\boldsymbol{\nabla} \varphi \times \boldsymbol{\pi})-2 \kappa(\boldsymbol{E} \times \boldsymbol{\pi})] \\
& -\frac{\hbar^{2}}{8 m^{2}}\left[\boldsymbol{\nabla} \cdot \boldsymbol{\nabla}[(\boldsymbol{\omega} \times \boldsymbol{x}) \cdot \boldsymbol{\pi}]+\nabla_{k}(\boldsymbol{\omega} \times \boldsymbol{\pi})^{k}\right]-\frac{\hbar}{4 m^{2}} \boldsymbol{\sigma} \cdot[(\boldsymbol{\omega} \times \boldsymbol{\pi}) \times \boldsymbol{\pi}] \\
& +\frac{\hbar^{2}}{8 m^{2}} \boldsymbol{\nabla} \cdot \boldsymbol{\nabla}\left(\nabla_{0} \Phi_{\mathrm{G}}\right)+\frac{\hbar}{4 m^{2}} \boldsymbol{\sigma} \cdot\left[\boldsymbol{\nabla}\left(\nabla_{0} \Phi_{\mathrm{G}}\right) \times \boldsymbol{\pi}\right]-\boldsymbol{\omega} \cdot(\boldsymbol{x} \times \boldsymbol{\pi})-\frac{\hbar}{2} \boldsymbol{\sigma} \cdot \boldsymbol{\omega}+e \boldsymbol{\varphi}+\left(\nabla_{0} \Phi_{\mathrm{G}}\right) .
\end{aligned}
$$

It is important to emphasize that terms such as $\boldsymbol{\nabla} \cdot \boldsymbol{\nabla} \varphi$ and $\sigma^{k} \epsilon^{i j}{ }_{k}\left(\nabla_{i} \nabla_{j} \Phi_{\mathrm{G}}\right)$ become $\nabla^{2} \varphi$ and $\boldsymbol{\sigma} \cdot\left[\boldsymbol{\nabla} \times \boldsymbol{\nabla} \Phi_{\mathrm{G}}\right]=0$ only for Cartesian co-ordinates, due to scale functions of $\lambda^{k}(u)=1$ in the definition of the gradient operator. Clearly, this also implies that $\boldsymbol{R}=0$ under the same circumstance. Again, retaining only the leading-order Hermitian terms, we show that the high-energy approximation of the Hamiltonian is

$$
\begin{aligned}
H_{\mathrm{CT}} \approx & (1+\boldsymbol{a} \cdot \boldsymbol{x})\left[\left[\sqrt{|\boldsymbol{\pi}|^{2}+m^{2}}-\frac{q^{3}}{\sqrt{1+q^{2}}} \frac{\hbar}{2 m} \boldsymbol{\sigma} \cdot \boldsymbol{R}^{\prime}\right] \frac{(\boldsymbol{\alpha} \cdot \boldsymbol{\pi})}{|\boldsymbol{\pi}|}+\frac{\kappa e \hbar}{2 m} \beta(i \boldsymbol{\alpha} \cdot \boldsymbol{E}-\boldsymbol{\sigma} \cdot \boldsymbol{B})\right] \\
& -\boldsymbol{\omega} \cdot(\boldsymbol{x} \times \boldsymbol{\pi})-\frac{\hbar}{2} \boldsymbol{\sigma} \cdot \boldsymbol{\omega}+e \varphi+\boldsymbol{\alpha} \cdot \boldsymbol{\nabla} \Phi_{\mathrm{G}}+\left(\nabla_{0} \Phi_{\mathrm{G}}\right) \\
& +\frac{q}{2|\boldsymbol{\pi}|}(1+\boldsymbol{a} \cdot \boldsymbol{x}) \frac{\kappa e \hbar}{2 m}\left[\hbar\left(\nabla_{k} E^{k}\right)+2 \boldsymbol{\sigma} \cdot(\boldsymbol{E} \times \boldsymbol{\pi})-\frac{2 \hbar}{|\boldsymbol{\pi}|^{2}} \boldsymbol{R}^{\prime} \cdot(\boldsymbol{E} \times \boldsymbol{\pi})-2\left(1-\frac{\hbar}{|\boldsymbol{\pi}|^{2}} \boldsymbol{\sigma} \cdot \boldsymbol{R}^{\prime}\right) \boldsymbol{B} \cdot \boldsymbol{\pi}\right] \\
& +\frac{q}{2|\boldsymbol{\pi}|}\left[-\frac{\hbar}{|\boldsymbol{\pi}|} \sqrt{|\boldsymbol{\pi}|^{2}+m^{2}}\left(1-\frac{\hbar}{|\boldsymbol{\pi}|^{2}} \boldsymbol{\sigma} \cdot \boldsymbol{R}^{\prime}\right) \sigma^{k} \epsilon_{j k}^{i} \nabla_{i}(\boldsymbol{a} \cdot \boldsymbol{x}) \pi^{j}+\frac{\hbar^{2}}{|\boldsymbol{\pi}|^{2}} \boldsymbol{\alpha} \cdot\left[\boldsymbol{R}^{\prime} \times \boldsymbol{\nabla}((\boldsymbol{\omega} \times \boldsymbol{x}) \cdot \boldsymbol{\pi})\right]\right. \\
& -\frac{\hbar^{2}}{2} \alpha^{k} \epsilon_{j k}^{i}\left(\nabla_{i} \omega^{j}\right)+\frac{\hbar^{2}}{|\boldsymbol{\pi}|^{2}}\left[\left(\boldsymbol{R}^{\prime} \cdot \boldsymbol{\omega}\right) \boldsymbol{\alpha} \cdot \boldsymbol{\pi}-\alpha^{j} R^{\prime k} \omega_{j} \pi_{k}\right] \\
& \left.+2\left(1-\frac{\hbar}{|\boldsymbol{\pi}|^{2}} \boldsymbol{\sigma} \cdot \boldsymbol{R}^{\prime}\right)\left[\nabla_{\mathrm{G}} \cdot \boldsymbol{\pi}-\frac{\hbar^{2}}{2}\left(\nabla_{k} a^{k}\right)\right]\right] \beta
\end{aligned}
$$

where $R^{\prime k}=R^{k}-e \epsilon^{k i}{ }_{j}\left(\nabla_{i} A^{j}\right)$.

\footnotetext{
${ }^{4}$ For notational purposes, the indices are left uncaretted for the remainder of this paper.
} 


\section{Spin-Flip Transition Rate}

The helicity operator $h$ is given by

$$
h \equiv \frac{\sigma \cdot \pi}{|\boldsymbol{\pi}|}
$$

and the spin-flip transition rate is

$$
\frac{d h}{d t}=\frac{i}{\hbar}[H, h]
$$

From (2.10) and (3.1), it is straightforward to show that the helicity precession operator is

$$
\begin{aligned}
|\boldsymbol{\pi}| \frac{d h}{d t}= & \boldsymbol{\sigma} \cdot\left[(\boldsymbol{\omega}+i \boldsymbol{a}) \times \boldsymbol{\pi}-\frac{\hbar}{2} \epsilon_{l m}^{k}\left[\left(\nabla_{k} a^{l}\right)-i\left(\nabla_{k} \omega^{l}\right)\right] \hat{\boldsymbol{x}}^{m}+(\boldsymbol{\omega} \times \boldsymbol{x}) \times \boldsymbol{R}+\epsilon_{k l m}\left[\boldsymbol{\nabla}\left(\omega^{l} x^{m}\right)\right] \pi^{k}\right. \\
& \left.-e(\boldsymbol{\omega} \times \boldsymbol{x})^{k}\left[\left(\nabla_{k} A_{l}\right)-\left(\nabla_{l} A_{k}\right)\right] \hat{\boldsymbol{x}}^{l}-e \boldsymbol{\nabla} \varphi-\nabla\left(\nabla_{0} \Phi_{\mathrm{G}}\right)\right] \\
& -\boldsymbol{\sigma} \cdot\left[\frac{2}{\hbar} \nabla \Phi_{\mathrm{G}} \times \boldsymbol{\pi}+i \boldsymbol{\nabla}(\boldsymbol{a} \cdot \boldsymbol{x}) \times \boldsymbol{\pi}+i \epsilon_{m}^{k l}{ }_{m}\left(\nabla_{k} \nabla_{l} \Phi_{\mathrm{G}}\right) \hat{\boldsymbol{x}}^{m}\right] \gamma^{5} \\
& +\boldsymbol{\sigma} \cdot\left[-m \boldsymbol{\nabla}(\boldsymbol{a} \cdot \boldsymbol{x})+\frac{\kappa e \hbar}{2 m}\left[\frac{2}{\hbar}(1+\boldsymbol{a} \cdot \boldsymbol{x}) \boldsymbol{B} \times \boldsymbol{\pi}+i(1+\boldsymbol{a} \cdot \boldsymbol{x}) \epsilon_{l m}^{k}\left(\nabla_{k} B^{l}\right) \hat{\boldsymbol{x}}^{m}+i \boldsymbol{\nabla}(\boldsymbol{a} \cdot \boldsymbol{x}) \times \boldsymbol{B}\right]\right] \beta \\
& -\frac{\kappa e \hbar}{2 m} \boldsymbol{\sigma} \cdot\left[\boldsymbol{\nabla}(\boldsymbol{a} \cdot \boldsymbol{x}) \times \boldsymbol{E}+(1+\boldsymbol{a} \cdot \boldsymbol{x}) \epsilon_{l m}^{k}\left(\nabla_{k} E^{l}\right) \hat{\boldsymbol{x}}^{m}-\frac{2 i}{\hbar}(1+\boldsymbol{a} \cdot \boldsymbol{x}) \boldsymbol{E} \times \boldsymbol{\pi}\right] \gamma^{5} \beta \\
& +\frac{\hbar}{2}\left[\left(\nabla_{k} \omega^{k}\right)+i\left(\nabla_{k} a^{k}\right)\right]-\left[\boldsymbol{\nabla}(\boldsymbol{a} \cdot \boldsymbol{x}) \cdot \boldsymbol{\pi}+\boldsymbol{\nabla} \cdot \boldsymbol{\nabla} \Phi_{\mathrm{G}}\right] \gamma^{5} \\
& +\frac{\kappa e \hbar}{2 m}\left[\boldsymbol{\nabla}(\boldsymbol{a} \cdot \boldsymbol{x}) \cdot \boldsymbol{B}+(1+\boldsymbol{a} \cdot \boldsymbol{x})\left(\nabla_{k} B^{k}\right)\right] \beta+\frac{i \kappa e \hbar}{2 m}\left[\boldsymbol{\nabla}(\boldsymbol{a} \cdot \boldsymbol{x}) \cdot \boldsymbol{E}+(1+\boldsymbol{a} \cdot \boldsymbol{x})\left(\nabla_{k} E^{k}\right)\right] \gamma^{5} \beta
\end{aligned}
$$

where $\hat{\boldsymbol{x}}^{m}$ is a unit vector of the spatial triad corresponding to the general curvilinear co-ordinate system.

It is a straightforward process to evaluate $d h_{\mathrm{FW}} / d t$ and $d h_{\mathrm{CT}} / d t$ using the Hamiltonians (2.26) and (2.27). However, to do this first requires that $h$ gets converted into an equivalent form using the FW and CT transformations, respectively, given that

$$
\frac{d}{d t} h_{\mathrm{FW} / \mathrm{CT}}=e^{i S_{\mathrm{FW} / \mathrm{CT}}} \frac{d h}{d t} e^{-i S_{\mathrm{FW} / \mathrm{CT}}}=e^{i S_{\mathrm{FW} / \mathrm{CT}}} \frac{i}{\hbar}[H, h] e^{-i S_{\mathrm{FW} / \mathrm{CT}}}=\frac{i}{\hbar}\left[H_{\mathrm{FW} / \mathrm{CT}}, h_{\mathrm{FW} / \mathrm{CT}}\right] .
$$

Therefore, the transformed versions of the helicity operator are

$$
\begin{aligned}
h_{\mathrm{FW}} & \approx \frac{1}{|\boldsymbol{\pi}|}\left\{\boldsymbol{\sigma} \cdot \boldsymbol{\pi}-\frac{1}{8 m^{2}}[\mathcal{O},[\mathcal{O}, \boldsymbol{\sigma} \cdot \boldsymbol{\pi}]]\right\} \\
h_{\mathrm{CT}} & \approx \frac{\boldsymbol{\sigma} \cdot \boldsymbol{\pi}}{|\boldsymbol{\pi}|}=h,
\end{aligned}
$$

where $\mathcal{O}$ is the set of odd operators which comprise the original Hamiltonian.

For the low-energy approximation, it is shown that the spin-flip transition rate is

$$
\frac{d}{d t} h_{\mathrm{FW}} \approx \frac{i}{\hbar}\left[H_{\mathrm{FW}}, h\right]+\frac{i}{\hbar}\left[H_{\mathrm{FW}}, h_{1}\right]
$$


where $h_{1}=-\left(1 / 8 m^{2}\right)[\mathcal{O},[\mathcal{O}, h]]$, and

$$
\begin{aligned}
& |\boldsymbol{\pi}| \frac{i}{\hbar}\left[H_{\mathrm{FW}}, h\right] \approx-(1+\boldsymbol{a} \cdot \boldsymbol{x})\left[\frac{1}{2 m} \boldsymbol{\sigma} \cdot \boldsymbol{\nabla}(\boldsymbol{\pi} \cdot \boldsymbol{\pi})+\frac{\hbar}{2 m}\left[\left(\nabla_{k} R^{\prime k}\right)+i \sigma^{k} \epsilon_{j k}^{i}\left(\nabla_{i} R^{\prime j}\right)\right]+\frac{1}{m} \boldsymbol{\sigma} \cdot\left(\boldsymbol{R}^{\prime} \times \boldsymbol{\pi}\right)\right] \beta \\
& -\boldsymbol{\sigma} \cdot \boldsymbol{\nabla}(\boldsymbol{a} \cdot \boldsymbol{x})\left[m+\frac{1}{2 m} \boldsymbol{\pi} \cdot \boldsymbol{\pi}\right] \beta+\frac{\kappa e}{m} \boldsymbol{\sigma} \cdot(\boldsymbol{B} \times \boldsymbol{\pi}) \beta+\frac{\kappa e \hbar}{2 m}\left[\left(\nabla_{k} B^{k}\right)+i \sigma^{k} \epsilon^{i}{ }_{j k}\left(\nabla_{i} B^{j}\right)\right] \beta \\
& -\frac{\hbar}{2 m}\left[\boldsymbol{\nabla}(\boldsymbol{a} \cdot \boldsymbol{x}) \cdot \boldsymbol{R}^{\prime}+\sigma^{k} \epsilon_{k}^{i j}\left(\nabla_{i} \nabla_{j} \Phi_{\mathrm{G}}\right)+i \boldsymbol{\sigma} \cdot\left[\boldsymbol{\nabla}(\boldsymbol{a} \cdot \boldsymbol{x}) \times \boldsymbol{R}^{\prime}\right]+i \sigma^{j} \nabla^{i}\left[\nabla_{i} \nabla_{j}-\nabla_{j} \nabla_{i}\right] \Phi_{\mathrm{G}}\right] \beta \\
& -\frac{1}{2 m}\left[\boldsymbol{\sigma} \cdot\left[(\boldsymbol{\nabla}(\boldsymbol{a} \cdot \boldsymbol{x}) \times \boldsymbol{\pi}) \times \boldsymbol{\pi}+2 \boldsymbol{\nabla}\left(\boldsymbol{\nabla} \Phi_{\mathrm{G}} \cdot \boldsymbol{\pi}\right)\right]+2 \sigma^{j}\left(\left[\nabla_{i} \nabla_{j}-\nabla_{j} \nabla_{i}\right] \Phi_{\mathrm{G}}\right) \pi^{i}\right] \beta \\
& +\frac{\hbar}{4 m}\left[-\left[\nabla_{k}(\boldsymbol{\nabla}(\boldsymbol{a} \cdot \boldsymbol{x}) \times \boldsymbol{\pi})^{k}\right]+\hbar \boldsymbol{\sigma} \cdot \boldsymbol{\nabla}\left(\nabla_{k} a^{k}\right)-\frac{\hbar}{2} \boldsymbol{\sigma} \cdot \boldsymbol{\nabla}(\boldsymbol{\nabla} \cdot \boldsymbol{\nabla}(\boldsymbol{a} \cdot \boldsymbol{x}))-i \sigma^{k} \epsilon_{j k}^{i}\left[\nabla_{i}(\boldsymbol{\nabla}(\boldsymbol{a} \cdot \boldsymbol{x}) \times \boldsymbol{\pi})^{j}\right]\right] \beta \\
& +\frac{\hbar^{2}}{8 m^{2}} \boldsymbol{\sigma} \cdot \boldsymbol{\nabla}\left[\boldsymbol{\nabla} \cdot \boldsymbol{\nabla}[(\boldsymbol{\omega} \times \boldsymbol{x}) \cdot \boldsymbol{\pi}]+\left[\nabla_{k}(\boldsymbol{\omega} \times \boldsymbol{\pi})^{k}\right]+e\left[\nabla_{k}\left(2 \kappa E^{k}+\nabla^{k} \varphi\right)\right]-\boldsymbol{\nabla} \cdot \boldsymbol{\nabla}\left(\nabla_{0} \Phi_{\mathrm{G}}\right)\right] \\
& +\frac{\hbar}{4 m^{2}}\left[\left[\nabla_{k}\left[(\boldsymbol{\omega} \times \boldsymbol{\pi}) \times \boldsymbol{\pi}+e(2 \kappa \boldsymbol{E}-\boldsymbol{\nabla} \varphi) \times \boldsymbol{\pi}-\boldsymbol{\nabla}\left(\nabla_{0} \Phi_{\mathrm{G}}\right) \times \boldsymbol{\pi}\right]^{k}\right]\right. \\
& \left.+i \sigma^{k} \epsilon_{j k}^{i}\left[\nabla_{i}\left[(\boldsymbol{\omega} \times \boldsymbol{\pi}) \times \boldsymbol{\pi}+e(2 \kappa \boldsymbol{E}-\boldsymbol{\nabla} \varphi) \times \boldsymbol{\pi}-\boldsymbol{\nabla}\left(\nabla_{0} \Phi_{\mathrm{G}}\right) \times \boldsymbol{\pi}\right]^{j}\right]\right] \\
& +\frac{1}{2 m^{2}} \sigma^{k} \epsilon_{i j k}\left[(\boldsymbol{\omega} \times \boldsymbol{\pi}) \times \boldsymbol{\pi}+e(2 \kappa \boldsymbol{E}-\boldsymbol{\nabla} \varphi) \times \boldsymbol{\pi}-\boldsymbol{\nabla}\left(\nabla_{0} \Phi_{\mathrm{G}}\right) \times \boldsymbol{\pi}\right]^{i} \pi^{j} \\
& +\boldsymbol{\sigma} \cdot[(\boldsymbol{\omega} \times \boldsymbol{x}) \times \boldsymbol{R}]-e \sigma^{j}(\boldsymbol{\omega} \times \boldsymbol{x})^{i}\left[\left(\nabla_{i} A_{j}\right)-\left(\nabla_{j} A_{i}\right)\right]+\frac{\hbar}{2}\left[\left(\nabla_{k} \omega^{k}\right)+i \sigma^{k} \epsilon_{j k}^{i}\left(\nabla_{i} \omega^{j}\right)\right] \\
& +\boldsymbol{\sigma} \cdot(\boldsymbol{\omega} \times \boldsymbol{\pi})+\epsilon_{k l m}\left[\boldsymbol{\sigma} \cdot \boldsymbol{\nabla}\left(\omega^{l} x^{m}\right)\right] \pi^{k}-\boldsymbol{\sigma} \cdot \boldsymbol{\nabla}\left(e \varphi+\left(\nabla_{0} \Phi_{\mathrm{G}}\right)\right) \\
& |\boldsymbol{\pi}| \frac{i}{\hbar}\left[H_{\mathrm{FW}}, h_{1}\right] \approx-\frac{\hbar^{2}}{8 m^{2}}\left[\sigma^{k}(\boldsymbol{\omega} \times \boldsymbol{x})^{j}\left[\nabla_{j} \nabla_{k}\left(\nabla_{i} \pi^{i}\right)\right]-\boldsymbol{\sigma} \cdot\left[\boldsymbol{\omega} \times \nabla\left(\nabla_{k} \pi^{k}\right)\right]\right] \\
& +\frac{\hbar^{2}}{4 m^{2}}\left[-(\boldsymbol{\omega} \times \boldsymbol{x}) \cdot \nabla\left[\sigma^{k} \nabla^{j}\left[N_{j k}+e\left(\nabla_{j} A_{k}\right)-e\left(\nabla_{k} A_{j}\right)\right]\right]\right. \\
& \left.-\sigma^{k} \epsilon_{i j k} \omega^{i}\left[\left(\nabla_{m} N^{m j}\right)+e\left(\nabla_{m} \nabla^{m} A^{j}\right)-e\left(\nabla_{m} \nabla^{j} A^{m}\right)\right]\right] \\
& +\frac{i \hbar^{2}}{4 m^{2}}\left[(\boldsymbol{\omega} \times \boldsymbol{x}) \cdot \boldsymbol{\nabla}\left[\nabla_{k}\left[R^{k}-e \epsilon_{j}^{i k}\left(\nabla_{i} A^{j}\right)\right]\right]+\left[N^{m n}+e\left(\nabla^{m} A^{n}\right)-e\left(\nabla^{n} A^{m}\right)\right]\left(\nabla_{m} \omega_{n}\right)\right. \\
& \left.-i \sigma^{k} \epsilon_{i j k}\left[N^{m i}+e\left(\nabla^{m} A^{i}\right)-e\left(\nabla^{i} A^{m}\right)\right]\left(\nabla_{m} \omega^{j}\right)\right] \\
& -\frac{i \hbar^{2}}{2 m^{2}}\left[\sigma^{k}(\boldsymbol{\omega} \times \boldsymbol{x}) \cdot \boldsymbol{\nabla}\left[\left[N_{m k}+e\left(\nabla_{m} A_{k}\right)-e\left(\nabla_{k} A_{m}\right)\right] \pi^{m}\right]\right. \\
& \left.-\sigma^{k} \epsilon_{i j k} \omega^{i}\left[N^{m j}+e\left(\nabla^{m} A^{j}\right)-e\left(\nabla^{j} A^{m}\right)\right] \pi_{m}\right] .
\end{aligned}
$$

In a similar fashion, for $H_{\mathrm{CT}}=H_{0}+q H_{1}$, the spin-flip transition rate for an ultra-relativistically moving spin- $1 / 2$ particle 
follows from (3.4), where

$$
\begin{aligned}
& |\boldsymbol{\pi}| \frac{i}{\hbar}\left[H_{0}, h\right]=-\frac{1}{|\boldsymbol{\pi}|} \sqrt{|\boldsymbol{\pi}|^{2}+m^{2}}[\boldsymbol{\nabla}(\boldsymbol{a} \cdot \boldsymbol{x}) \cdot \boldsymbol{\pi}+i \boldsymbol{\sigma} \cdot[\boldsymbol{\nabla}(\boldsymbol{a} \cdot \boldsymbol{x}) \times \boldsymbol{\pi}]] \gamma^{5} \\
& +\frac{q^{3}}{\sqrt{1+q^{2}}} \frac{\hbar}{2 m} \frac{1}{|\boldsymbol{\pi}|}\left[[\boldsymbol{\sigma} \cdot \boldsymbol{\nabla}(\boldsymbol{a} \cdot \boldsymbol{x})] \boldsymbol{R}^{\prime} \cdot \boldsymbol{\pi}+i[\boldsymbol{\nabla}(\boldsymbol{a} \cdot \boldsymbol{x})] \cdot\left(\boldsymbol{R}^{\prime} \times \boldsymbol{\pi}\right)-\boldsymbol{\sigma} \cdot\left[\boldsymbol{\nabla}(\boldsymbol{a} \cdot \boldsymbol{x}) \times\left(\boldsymbol{R}^{\prime} \times \boldsymbol{\pi}\right)\right]\right] \gamma^{5} \\
& +(1+\boldsymbol{a} \cdot \boldsymbol{x})\left[\frac { q ^ { 3 } } { \sqrt { 1 + q ^ { 2 } } } \frac { \hbar } { 2 m } \frac { 1 } { | \boldsymbol { \pi } | } \left[\left[\boldsymbol{\sigma} \cdot \boldsymbol{\nabla}\left(\boldsymbol{R}^{\prime} \cdot \boldsymbol{\pi}\right)\right]+\frac{2 i}{\hbar} \boldsymbol{\sigma} \cdot\left[\left(\boldsymbol{R}^{\prime} \times \boldsymbol{\pi}\right) \times \boldsymbol{\pi}\right]\right.\right. \\
& \left.\left.+i\left[\nabla_{k}\left(\boldsymbol{R}^{\prime} \times \boldsymbol{\pi}\right)^{k}\right]-\sigma^{k} \epsilon_{j k}^{i}\left[\nabla_{i}\left(\boldsymbol{R}^{\prime} \times \boldsymbol{\pi}\right)^{j}\right]\right]\right] \gamma^{5} \\
& +\frac{\kappa e \hbar}{2 m} \boldsymbol{\sigma} \cdot\left[\frac{2}{\hbar}(1+\boldsymbol{a} \cdot \boldsymbol{x}) \boldsymbol{B} \times \boldsymbol{\pi}+i(1+\boldsymbol{a} \cdot \boldsymbol{x}) \epsilon^{k}{ }_{l m}\left(\nabla_{k} B^{l}\right) \hat{\boldsymbol{x}}^{m}+i \boldsymbol{\nabla}(\boldsymbol{a} \cdot \boldsymbol{x}) \times \boldsymbol{B}\right] \beta \\
& -\frac{\kappa e \hbar}{2 m} \boldsymbol{\sigma} \cdot\left[\boldsymbol{\nabla}(\boldsymbol{a} \cdot \boldsymbol{x}) \times \boldsymbol{E}+(1+\boldsymbol{a} \cdot \boldsymbol{x}) \epsilon_{l m}^{k}\left(\nabla_{k} E^{l}\right) \hat{\boldsymbol{x}}^{m}-\frac{2 i}{\hbar}(1+\boldsymbol{a} \cdot \boldsymbol{x}) \boldsymbol{E} \times \boldsymbol{\pi}\right] \gamma^{5} \beta \\
& +\frac{\kappa e \hbar}{2 m}\left[\nabla(\boldsymbol{a} \cdot \boldsymbol{x}) \cdot \boldsymbol{B}+(1+\boldsymbol{a} \cdot \boldsymbol{x})\left(\nabla_{k} B^{k}\right)\right] \beta+\frac{i \kappa e \hbar}{2 m}\left[\boldsymbol{\nabla}(\boldsymbol{a} \cdot \boldsymbol{x}) \cdot \boldsymbol{E}+(1+\boldsymbol{a} \cdot \boldsymbol{x})\left(\nabla_{k} E^{k}\right)\right] \gamma^{5} \beta \\
& -\boldsymbol{\sigma} \cdot\left[\frac{2}{\hbar} \nabla \Phi_{\mathrm{G}} \times \boldsymbol{\pi}+i \epsilon_{m}^{k l}\left(\nabla_{k} \nabla_{l} \Phi_{\mathrm{G}}\right) \hat{\boldsymbol{x}}^{m}\right] \gamma^{5}+\frac{\hbar}{2}\left(\nabla_{k} \omega^{k}\right)-\nabla \cdot \nabla \Phi_{\mathrm{G}} \gamma^{5} \\
& +\boldsymbol{\sigma} \cdot\left[\boldsymbol{\omega} \times \boldsymbol{\pi}+\frac{i \hbar}{2} \epsilon_{l m}^{k}\left(\nabla_{k} \omega^{l}\right) \hat{\boldsymbol{x}}^{m}+(\boldsymbol{\omega} \times \boldsymbol{x}) \times \boldsymbol{R}+\epsilon_{k l m}\left[\nabla\left(\omega^{l} x^{m}\right)\right] \pi^{k}\right. \\
& \left.-e(\boldsymbol{\omega} \times \boldsymbol{x})^{k}\left[\left(\nabla_{k} A_{l}\right)-\left(\nabla_{l} A_{k}\right)\right] \hat{\boldsymbol{x}}^{l}-e \boldsymbol{\nabla} \varphi-\nabla\left(\nabla_{0} \Phi_{\mathrm{G}}\right)\right] \\
& |\boldsymbol{\pi}| \frac{i}{\hbar}\left[H_{1}, h\right]=-\frac{1}{|\boldsymbol{\pi}|} \frac{\kappa e \hbar}{2 m}[\boldsymbol{\nabla}(\boldsymbol{a} \cdot \boldsymbol{x}) \cdot(\boldsymbol{E} \times \boldsymbol{\pi})+i \boldsymbol{\sigma} \cdot[\boldsymbol{\nabla}(\boldsymbol{a} \cdot \boldsymbol{x}) \times(\boldsymbol{E} \times \boldsymbol{\pi})]-[\boldsymbol{\sigma} \cdot \boldsymbol{\nabla}(\boldsymbol{a} \cdot \boldsymbol{x})] \boldsymbol{B} \cdot \boldsymbol{\pi}] \\
& +\frac{1}{|\boldsymbol{\pi}|}(1+\boldsymbol{a} \cdot \boldsymbol{x}) \frac{\kappa e \hbar}{2 m}\left[-\left[\left[\nabla_{k}(\boldsymbol{E} \times \boldsymbol{\pi})^{k}\right]+i \sigma^{k} \epsilon_{j k}^{i}\left[\nabla_{i}(\boldsymbol{E} \times \boldsymbol{\pi})^{j}\right]\right]-\frac{2}{\hbar} \boldsymbol{\sigma} \cdot[(\boldsymbol{E} \times \boldsymbol{\pi}) \times \boldsymbol{\pi}]+\boldsymbol{\sigma} \cdot \boldsymbol{\nabla}(\boldsymbol{B} \cdot \boldsymbol{\pi})\right] \\
& +\frac{1}{2|\boldsymbol{\pi}|}\left[\frac{\hbar}{|\boldsymbol{\pi}|} \sqrt{|\boldsymbol{\pi}|^{2}+m^{2}}\left[\left[\nabla_{k}(\boldsymbol{\nabla}(\boldsymbol{a} \cdot \boldsymbol{x}) \times \boldsymbol{\pi})^{k}\right]+\frac{2}{\hbar} \boldsymbol{\sigma} \cdot[(\boldsymbol{\nabla}(\boldsymbol{a} \cdot \boldsymbol{x}) \times \boldsymbol{\pi}) \times \boldsymbol{\pi}]+i \sigma^{k} \epsilon_{j k}^{i}\left[\nabla_{i}(\boldsymbol{\nabla}(\boldsymbol{a} \cdot \boldsymbol{x}) \times \boldsymbol{\pi})^{j}\right]\right]\right. \\
& +\frac{2 \hbar}{|\boldsymbol{\pi}|^{2}}\left[\left(\nabla_{k} R^{\prime k}\right)+i \sigma^{k} \epsilon_{j k}^{i}\left(\nabla_{i} R^{\prime j}\right)+\frac{2}{\hbar} \boldsymbol{\sigma} \cdot\left(\boldsymbol{R}^{\prime} \times \boldsymbol{\pi}\right)\right] \boldsymbol{\nabla} \Phi_{\mathrm{G}} \cdot \boldsymbol{\pi}-2 \boldsymbol{\sigma} \cdot \boldsymbol{\nabla}\left(\boldsymbol{\nabla} \Phi_{\mathrm{G}} \cdot \boldsymbol{\pi}\right) \\
& \left.+\frac{2 \hbar}{|\boldsymbol{\pi}|^{2}}\left[\boldsymbol{R}^{\prime} \cdot \boldsymbol{\nabla}\left(\boldsymbol{\nabla} \Phi_{\mathrm{G}} \cdot \boldsymbol{\pi}\right)+i \boldsymbol{\sigma} \cdot\left[\boldsymbol{R}^{\prime} \times \boldsymbol{\nabla}\left(\boldsymbol{\nabla} \Phi_{\mathrm{G}} \cdot \boldsymbol{\pi}\right)\right]\right]\right] \beta
\end{aligned}
$$

After neglecting the non-Hermitian term $-(i \hbar / 2)(\boldsymbol{\alpha} \cdot \boldsymbol{a})$ in (2.10), it is straightforward to confirm that (3.9) and (3.10) reduce to (3.3) in the limit as $q \rightarrow 0$.

\section{Spin Evolution}

It is useful to evaluate the spin evolution of a spin-1/2 particle in non-inertial motion, such as that due to a circular orbit in an ideal storage ring. This requires that the operator expressions for the helicity transition rate must be converted 
into amplitudes and projected into the laboratory frame. Suppose that a beam of spin- $1 / 2$ particles follows a circular orbit in an idealized storage ring, allowing for vertical and horizontal fluctuations about the beam's mean trajectory. Then, by adopting cylindrical co-ordinates $(r, \theta, z, \tau)$ to describe an accelerated frame tangent to the beam orbit, where

$$
P^{1}=-i \hbar \frac{\partial}{\partial r}, \quad P^{2}=\frac{-i \hbar}{r} \frac{\partial}{\partial \theta}, \quad P^{3}=-i \hbar \frac{\partial}{\partial z},
$$

it follows that $\boldsymbol{R}=\left(0,0,-\frac{1}{r} P^{2}\right)$. Assuming a mean orbital radius of $r_{0}$ and orbital frequency of $\omega_{0}$ in the laboratory frame, the relationship between the accelerated frame and the laboratory frame in Cartesian co-ordinates $(x, y, z, t)$, whose origin is at the centre of the storage ring, is given by [8, 36

$$
\begin{aligned}
x & =\left(r_{0}+\delta r\right) \cos \left(\gamma \omega_{0} \tau\right)-\gamma\left(r_{0} \delta \theta\right) \sin \left(\gamma \omega_{0} \tau\right) \\
y & =\left(r_{0}+\delta r\right) \sin \left(\gamma \omega_{0} \tau\right)+\gamma\left(r_{0} \delta \theta\right) \cos \left(\gamma \omega_{0} \tau\right) \\
t & =\gamma\left(\tau+r_{0}^{2} \omega_{0} \delta \theta\right),
\end{aligned}
$$

where $\delta r$ and $\delta \theta$ are the radial and angular fluctuations about the mean orbit, $\gamma=\left(1-\omega_{0}^{2} r_{0}^{2}\right)^{-1 / 2}$, and $\tau$ is the proper time.

To obtain the spin-flip transition amplitude, it is necessary to adopt the Dirac representation and evaluate it in the instantaneous rest frame of the particle. Therefore, given the corresponding ket vectors 38.

$$
\begin{aligned}
& |+\rangle_{\text {up }} \equiv\left(\begin{array}{c}
\left(\begin{array}{l}
1 \\
0
\end{array}\right) \\
\left(\begin{array}{l}
0 \\
0
\end{array}\right)
\end{array}\right) \quad|-\rangle_{\text {up }} \equiv\left(\begin{array}{c}
\left(\begin{array}{l}
0 \\
1
\end{array}\right) \\
\left(\begin{array}{l}
0 \\
0
\end{array}\right)
\end{array}\right) \\
& |+\rangle_{\mathrm{dn}} \equiv\left(\begin{array}{c}
\left(\begin{array}{l}
0 \\
0
\end{array}\right) \\
\left(\begin{array}{l}
1 \\
0
\end{array}\right)
\end{array}\right) \quad|-\rangle_{\mathrm{dn}} \equiv\left(\begin{array}{c}
\left(\begin{array}{l}
0 \\
0
\end{array}\right) \\
\left(\begin{array}{l}
0 \\
1
\end{array}\right)
\end{array}\right) \text {, }
\end{aligned}
$$

it is shown that the spin-flip transition amplitude due to some quantum operator $Q$ is

$$
\langle Q\rangle \equiv\langle\mp|Q| \pm\rangle_{\mathrm{up} / \mathrm{dn}} .
$$

Then for some arbitrary spin-independent quantum number $\boldsymbol{K}$ coupled to $\boldsymbol{\sigma}$, it follows that

$$
\begin{aligned}
\langle(\boldsymbol{\sigma} \cdot \boldsymbol{K})\rangle & =\left(\hat{\boldsymbol{x}}^{1} \pm i \hat{\boldsymbol{x}}^{2}\right) \cdot \boldsymbol{K} \\
\langle(\boldsymbol{\sigma} \cdot \boldsymbol{K}) \beta\rangle & = \pm\left(\hat{\boldsymbol{x}}^{1} \pm i \hat{\boldsymbol{x}}^{2}\right) \cdot \boldsymbol{K},
\end{aligned}
$$

where all other matrix elements vanish, and the overall \pm in (4.6) denotes the sign of the contribution specific to the up/dn state, respectively.

It is straightforward to verify [12] that the unit vectors $\hat{\boldsymbol{x}}^{1}(\tau)$ and $\hat{\boldsymbol{x}}^{2}(\tau)$ are related to the time-independent Cartesian unit vectors $\hat{\boldsymbol{x}}$ and $\hat{\boldsymbol{y}}$ in the laboratory frame by

$$
\hat{\boldsymbol{x}}^{1}(\tau) \pm i \hat{\boldsymbol{x}}^{2}(\tau)=(\hat{\boldsymbol{x}} \pm i \hat{\boldsymbol{y}}) e^{\mp i \gamma \omega_{0} \tau}=(\hat{\boldsymbol{x}} \pm i \hat{\boldsymbol{y}}) e^{\mp i \omega_{0} t} .
$$


With (4.5) and (4.6), it is formally shown that the rate of change of the spin-flip transition is

$$
\left\langle\frac{d h(t)}{d t}\right\rangle=\frac{1}{|\boldsymbol{\pi}|}\left(\hat{\boldsymbol{x}}^{1}(t) \pm i \hat{\boldsymbol{x}}^{2}(t)\right) \cdot\left[\boldsymbol{\Lambda}^{0} \pm \boldsymbol{\Lambda}^{1}\right]=\frac{1}{|\boldsymbol{\pi}|}(\hat{\boldsymbol{x}} \pm i \hat{\boldsymbol{y}}) \cdot\left[\boldsymbol{\Lambda}^{0} \pm \boldsymbol{\Lambda}^{1}\right] e^{\mp i \omega_{0} t},
$$

where $\boldsymbol{\Lambda}^{0}$ and $\boldsymbol{\Lambda}^{1}$ are the amplitudes corresponding to (4.5) and (4.6), respectively. It then follows that the spin evolution is

$$
\begin{aligned}
\langle h(t)\rangle & =\frac{1}{|\boldsymbol{\pi}|}(\hat{\boldsymbol{x}} \pm i \hat{\boldsymbol{y}}) \cdot \boldsymbol{\pi}+\int_{0}^{t}\left\langle\frac{d h\left(t^{\prime}\right)}{d t}\right\rangle d t^{\prime} \\
& =\frac{1}{|\boldsymbol{\pi}|}(\hat{\boldsymbol{x}} \pm i \hat{\boldsymbol{y}}) \cdot\left[\boldsymbol{\pi} \pm \frac{i}{\omega_{0}}\left(\boldsymbol{\Lambda}^{0} \pm \boldsymbol{\Lambda}^{1}\right)\left(e^{\mp i \omega_{0} t}-1\right)\right] .
\end{aligned}
$$

By evaluating (4.5) and (4.6) for each of the cases under consideration, the amplitudes corresponding to the Dirac Hamiltonian in its original representation, and also its FW- and CT-transformed counterparts, can be obtained. Therefore, from (3.3), it is shown that

$$
\begin{aligned}
\boldsymbol{\Lambda}_{\text {Dirac }}^{0}= & \boldsymbol{\omega} \times \boldsymbol{\pi}+\frac{i \hbar}{2} \epsilon_{j k}^{i}\left(\nabla_{i} \omega^{j}\right) \hat{\boldsymbol{x}}^{k}+(\boldsymbol{\omega} \times \boldsymbol{x}) \times \boldsymbol{R}+\epsilon_{k l m}\left[\nabla\left(\omega^{l} x^{m}\right)\right] \pi^{k} \\
& -e(\boldsymbol{\omega} \times \boldsymbol{x})^{k}\left[\left(\nabla_{k} A_{l}\right)-\left(\nabla_{l} A_{k}\right)\right] \hat{\boldsymbol{x}}^{l}-e \boldsymbol{\nabla} \varphi-\nabla\left(\nabla_{0} \Phi_{\mathrm{G}}\right) \\
\boldsymbol{\Lambda}_{\text {Dirac }}^{1}= & -m \boldsymbol{\nabla}(\boldsymbol{a} \cdot \boldsymbol{x})+\boldsymbol{\Lambda}_{\kappa}^{1} \\
\boldsymbol{\Lambda}_{\kappa}^{1}= & \frac{\kappa e \hbar}{2 m}\left[\frac{2}{\hbar}(1+\boldsymbol{a} \cdot \boldsymbol{x}) \boldsymbol{B} \times \boldsymbol{\pi}+i(1+\boldsymbol{a} \cdot \boldsymbol{x}) \epsilon_{j k}^{i}\left(\nabla_{i} B^{j}\right) \hat{\boldsymbol{x}}^{k}+i \boldsymbol{\nabla}(\boldsymbol{a} \cdot \boldsymbol{x}) \times \boldsymbol{B}\right],
\end{aligned}
$$

for the helicity evolution involving the original Dirac Hamiltonian, where $\boldsymbol{\omega}=\gamma^{2} \omega_{0} \hat{\boldsymbol{z}}$ [8] [37] in the rotating frame of reference. In an ideal storage ring, the corrections proportional to $\boldsymbol{a}$ found in 4.12) and several other expressions for $\Lambda$ given below are really of second order because $B=m \omega / e$. The latter relationship also ensures that $\Lambda_{\kappa}^{1}$ is mass independent. The remaining terms in (4.12) do not contribute to $g-2$ experiments because of geometrical constraints or because $B$ is uniform. They would contribute, however, to experiments where these constraints were relaxed.

The first and second terms in (4.10) originate from the Mashhoon coupling in the Hamiltonian and the second term would not occur in experiments with $\omega$ constant. The first and fourth term play an important role in $g-2$ experiments and are discussed below. The sixth term is well known and can also be found in the calculation of Sakurai [38. Together, the fifth and sixth terms form something akin to the Lorentz force in a rotating frame.

The third term vanishes in Cartesian coordinates because that case involves only linear momenta and the commutator in (2.17) therefore vanishes. However, when the coordinates are not Cartesian, the commutator of the momenta mixes linear momentum and angular momentum components and, in general, does not vanish. Its contribution to the precession equation is $\boldsymbol{\sigma} \cdot[(\boldsymbol{\omega} \times \boldsymbol{x}) \times \boldsymbol{R}]$ and has the same dimensions as the term $\boldsymbol{\sigma} \cdot(\boldsymbol{\omega} \times \boldsymbol{p})$ that appears in the Thomas-BMT equation [39]. The third term may be small for the geometry of $g-2$ experiments, but not so for other types of spin motion like those considered in spin rotators and Siberian snakes.

The last term in (4.10) is new and has interesting consequences. In fact, it follows from (4.8)-(4.12) that in the absence of electromagnetic potentials and in the limit as $m \rightarrow 0$,

$$
\left\langle\frac{d h}{d t}\right\rangle_{m=0}=-\frac{1}{|\boldsymbol{\pi}|}\left(\hat{\boldsymbol{x}}^{1} \pm i \hat{\boldsymbol{x}}^{2}\right) \cdot \nabla\left(\nabla_{0} \Phi_{\mathrm{G}}\right)
$$

where, for the case of a Cartesian co-ordinate frame,

$$
\nabla_{i}\left(\nabla_{0} \Phi_{\mathrm{G}}\right)=-\frac{1}{2}\left[\gamma_{00, i} p^{0}+\left(\gamma_{0 j, i}+\gamma_{i j, 0}-\gamma_{0 i, j}\right) p^{j}\right] .
$$


For instance, given a stationary metric of the type shown by Hehl and $\mathrm{Ni}$ [20, we obtain

$$
\begin{aligned}
\nabla_{i}\left(\nabla_{0} \Phi_{\mathrm{G}}\right) & =-(\boldsymbol{a} \cdot \boldsymbol{x})_{, i} p^{0}-\frac{1}{2}\left[\epsilon_{j k l}\left(\omega^{k} x^{l}\right)_{, i}-\epsilon_{i k l}\left(\omega^{k} x^{l}\right)_{, j}\right] p^{j} \\
& \approx a_{i} p^{0}+\epsilon_{i j k} \omega^{j} p^{k} \neq 0 .
\end{aligned}
$$

This result is somewhat surprising, but not unlike the electromagnetic case where the presence of an electric field also violates helicity conservation [38. The fact that $\dot{h} \neq 0$ here challenges commonly held views that the helicity is a constant of motion for massless particles 34. However, particles are known to acquire an effective mass when acted upon by inertia-gravitation. By multiplying (2.1) on the left by $\left(-i \gamma^{\nu}(x) D_{\nu}-m / \hbar\right)$ and using the relations $\left[D_{\mu}, D_{\nu}\right]=-i \sigma^{\alpha \beta} R_{\alpha \beta \mu \nu}, \sigma^{\mu \nu} \sigma^{a b} R_{\mu \nu \alpha \beta}=2 R$, where $R$ is the Ricci curvature scalar, we can obtain

$$
\left(g^{\mu \nu} D_{\mu} D_{\nu}-\frac{R}{4}+\frac{m^{2}}{\hbar^{2}}\right) \psi(x)=0 .
$$

Weyl [40] was the first one to suggest that $m_{\mathrm{eff}} \equiv \hbar \sqrt{(m / \hbar)^{2}-R / 4}$ behaves as an effective mass. Notice that $m_{\mathrm{eff}} \neq 0$ even when $m=0$, and that $R \neq 0$ when it represents, in its linearized form, pure inertia.

A second interesting result follows from the same equations. By choosing Cartesian co-ordinates so that the term $(\boldsymbol{\omega} \times \boldsymbol{x}) \times \boldsymbol{R}$ vanishes, dropping second order terms in $\omega$, and combining the remaining first and fourth terms in (4.10) with the term $(\kappa e / m) \boldsymbol{B} \times \boldsymbol{\pi}$ in (4.11), we obtain

$$
\left\langle\frac{d h}{d t}\right\rangle \simeq \frac{1}{|\boldsymbol{\pi}|}\left(\hat{\boldsymbol{x}}^{1} \pm i \hat{\boldsymbol{x}}^{2}\right) \cdot\left[ \pm \frac{\kappa e}{m} \boldsymbol{B} \times \boldsymbol{\pi}+\epsilon_{i j k}\left(\boldsymbol{\nabla} \omega^{i}\right) x^{j} \pi^{k}\right] .
$$

The Mashhoon term therefore disappears irrespective of whether $\omega$ is constant or not. The first term proportional to $\kappa$ and with $B=m \omega / e$ on the right hand side of (4.17) is the term normally measured in $g-2$ experiments. If $\omega$ is inhomogeneous, then the second term also contributes to the helicity precession. This term can then be neglected only for particular geometrical configurations of the parameters involved.

From (3.7) and (3.8), the corresponding expressions for the FW-transformed Hamiltonian are

$$
\begin{aligned}
\boldsymbol{\Lambda}_{\mathrm{FW}}^{0}= & \boldsymbol{\Lambda}_{\mathrm{Dirac}}^{0}+\frac{1}{2 m^{2}} \epsilon_{i j k}\left[(\boldsymbol{\omega} \times \boldsymbol{\pi}) \times \boldsymbol{\pi}+e(2 \kappa \boldsymbol{E}-\nabla \varphi) \times \boldsymbol{\pi}-\boldsymbol{\nabla}\left(\nabla_{0} \Phi_{\mathrm{G}}\right) \times \boldsymbol{\pi}\right]^{i} \pi^{j} \hat{\boldsymbol{x}}^{k} \\
& +\frac{\hbar^{2}}{8 m^{2}}\left[\boldsymbol{\nabla}\left[\boldsymbol{\nabla} \cdot \boldsymbol{\nabla}[(\boldsymbol{\omega} \times \boldsymbol{x}) \cdot \boldsymbol{\pi}]+\left[\nabla_{k}(\boldsymbol{\omega} \times \boldsymbol{\pi})^{k}\right]+e\left[\nabla_{k}\left(2 \kappa E^{k}+\nabla^{k} \varphi\right)\right]-\boldsymbol{\nabla} \cdot \boldsymbol{\nabla}\left(\nabla_{0} \Phi_{\mathrm{G}}\right)\right]\right. \\
& \left.-\left[(\boldsymbol{\omega} \times \boldsymbol{x})^{j}\left[\nabla_{j} \nabla_{k}\left(\nabla_{i} \pi^{i}\right)\right] \hat{\boldsymbol{x}}^{k}-\boldsymbol{\omega} \times\left[\boldsymbol{\nabla}\left(\nabla_{k} \pi^{k}\right)\right]\right]\right] \\
& +\frac{\hbar^{2}}{4 m^{2}}\left[(\boldsymbol{\omega} \times \boldsymbol{x}) \cdot \boldsymbol{\nabla}\left[\left(\nabla_{1} R^{3}\right) \hat{\boldsymbol{x}}^{2}-\left(\nabla_{2} R^{3}\right) \hat{\boldsymbol{x}}^{1}-e\left(\left(\nabla^{j} \nabla_{j} A_{k}\right)-\left(\nabla^{j} \nabla_{k} A_{j}\right)\right) \hat{\boldsymbol{x}}^{k}\right]\right. \\
& +\left[R^{3}\left[\epsilon^{2}{ }_{j k}\left(\nabla_{1} \omega^{j}\right)-\epsilon^{1}{ }_{j k}\left(\nabla_{2} \omega^{j}\right)\right]+\epsilon_{i j k} e\left(\left(\nabla_{m} A^{i}\right)-\left(\nabla^{i} A_{m}\right)\right)\left(\nabla^{m} \omega^{j}\right)\right] \hat{\boldsymbol{x}}^{k} \\
& \left.+\omega^{i}\left[\epsilon^{2}{ }_{i k}\left(\nabla_{1} R^{3}\right)-\epsilon^{1}{ }_{i k}\left(\nabla_{2} R^{3}\right)+\epsilon_{i j k} e\left(\left(\nabla^{m} \nabla_{m} A^{j}\right)-\left(\nabla^{m} \nabla^{j} A_{m}\right)\right)\right] \hat{\boldsymbol{x}}^{k}\right] \\
& +\frac{i \hbar}{4 m^{2}} \epsilon^{i}{ }_{j k} \nabla_{i}\left[(\boldsymbol{\omega} \times \boldsymbol{\pi}) \times \boldsymbol{\pi}+e(2 \kappa \boldsymbol{E}-\boldsymbol{\nabla} \varphi) \times \boldsymbol{\pi}-\left[\nabla\left(\nabla_{0} \Phi_{\mathrm{G}}\right)\right] \times \boldsymbol{\pi}\right]^{j} \hat{\boldsymbol{x}}^{k} \\
& -\frac{i \hbar^{2}}{2 m^{2}}\left[(\boldsymbol{\omega} \times \boldsymbol{x}) \cdot \boldsymbol{\nabla}\left[R^{3}\left(\pi^{1} \hat{\boldsymbol{x}}^{2}-\pi^{2} \hat{\boldsymbol{x}}^{1}\right)+e\left[\left(\nabla_{j} A_{k}\right)-\left(\nabla_{k} A_{j}\right)\right] \pi^{j} \hat{\boldsymbol{x}}^{k}\right]\right.
\end{aligned}
$$




$$
\begin{aligned}
& \left.+\omega^{i}\left[R^{3}\left(\epsilon^{2}{ }_{i k} \pi^{1}-\epsilon^{1}{ }_{i k} \pi^{2}\right)+\epsilon_{i j k} e\left[\left(\nabla_{m} A^{j}\right)-\left(\nabla^{j} A_{m}\right)\right] \pi^{m}\right] \hat{\boldsymbol{x}}^{k}\right] \\
\boldsymbol{\Lambda}_{\mathrm{FW}}^{1}= & -(1+\boldsymbol{a} \cdot \boldsymbol{x})\left[\frac{1}{2 m} \boldsymbol{\nabla}(\boldsymbol{\pi} \cdot \boldsymbol{\pi})+\frac{1}{m} \boldsymbol{\sigma} \cdot\left(\boldsymbol{R}^{\prime} \times \boldsymbol{\pi}\right)+\frac{i \hbar}{2 m} \epsilon_{j k}^{i}\left(\nabla_{i} R^{j}\right) \hat{\boldsymbol{x}}^{k}\right] \\
& -\boldsymbol{\nabla}(\boldsymbol{a} \cdot \boldsymbol{x})\left[m+\frac{1}{2 m} \boldsymbol{\pi} \cdot \boldsymbol{\pi}\right]+\frac{\kappa e}{m} \boldsymbol{\sigma} \cdot(\boldsymbol{B} \times \boldsymbol{\pi})+\frac{i \kappa e \hbar}{2 m} \epsilon^{i}{ }_{j k}\left(\nabla_{i} B^{j}\right) \hat{\boldsymbol{x}}^{k}-\frac{\hbar}{2 m} \epsilon^{i j}\left(\nabla_{i} \nabla_{j} \Phi_{\mathrm{G}}\right) \hat{\boldsymbol{x}}^{k} \\
& -\frac{i \hbar}{2 m}\left[\boldsymbol{\nabla}(\boldsymbol{a} \cdot \boldsymbol{x}) \times \boldsymbol{R}^{\prime}+\left(\nabla^{j}\left[\nabla_{j} \nabla_{k}-\nabla_{k} \nabla_{j}\right] \Phi_{\mathrm{G}}\right) \hat{\boldsymbol{x}}^{k}\right] \\
& -\frac{1}{2 m}\left[(\boldsymbol{\nabla}(\boldsymbol{a} \cdot \boldsymbol{x}) \times \boldsymbol{\pi}) \times \boldsymbol{\pi}+2 \boldsymbol{\nabla}\left(\boldsymbol{\nabla} \Phi_{\mathrm{G}} \cdot \boldsymbol{\pi}\right)\right]-\frac{1}{m}\left(\left[\nabla_{j} \nabla_{k}-\nabla_{k} \nabla_{j}\right] \Phi_{\mathrm{G}}\right) \pi^{j} \hat{\boldsymbol{x}}^{k} \\
& -\frac{\hbar^{2}}{8 m} \boldsymbol{\nabla}(\boldsymbol{\nabla} \cdot \boldsymbol{\nabla}(\boldsymbol{a} \cdot \boldsymbol{x}))-\frac{i \hbar}{4 m} \epsilon_{j k}^{i}\left[\nabla_{i}(\boldsymbol{\nabla}(\boldsymbol{a} \cdot \boldsymbol{x}) \times \boldsymbol{\pi})^{j}\right] \hat{\boldsymbol{x}}^{k} .
\end{aligned}
$$

Again assuming a local Cartesian frame, the leading-order contributions to the amplitude in the low-energy approximation, of order $1 / \mathrm{m}$, come from the second and sixth terms of (4.19), which yield the total magnetic moment term $[(1+\kappa) e / m] \boldsymbol{\sigma} \cdot(\boldsymbol{B} \times \boldsymbol{\pi})$. Other noteworthy leading-order contributions are due to the eleventh term in (4.19), which come from the acceleration-induced spin-orbit coupling term first found by Hehl and Ni [20, 17, and also the twelfth and thirteenth terms due to the gravitational energy redshift term found by Singh and Papini [17. As for terms of order $1 / \mathrm{m}^{2}$, the leading contributions [17] are due to the second to fourth terms found in (4.18), namely the spin-orbit coupling from the Mashhoon effect, electric field, and gravitational field, respectively. In addition, the seventh and eighth terms of (4.18) identify contributions from the Darwin energy terms due to electromagnetism 38 and gravitation, also first found by Singh and Papini [17, and later by Obukhov [41. All other terms in (4.18) and (4.19) involving gradients of primarily small quantities can be safely regarded as negligible by comparison.

From (3.9) and (3.10), it is shown that the amplitudes for the CT-transformed Hamiltonian are

$$
\begin{aligned}
\boldsymbol{\Lambda}_{\mathrm{CT}}^{0}= & \boldsymbol{\Lambda}_{\mathrm{Dirac}}^{0}+\frac{1}{|\boldsymbol{\pi}|} \frac{\kappa e \hbar}{2 m}[\boldsymbol{\nabla}(\boldsymbol{a} \cdot \boldsymbol{x}) \boldsymbol{B} \cdot \boldsymbol{\pi}-i \boldsymbol{\nabla}(\boldsymbol{a} \cdot \boldsymbol{x}) \times(\boldsymbol{E} \times \boldsymbol{\pi}) \\
& \left.+(1+\boldsymbol{a} \cdot \boldsymbol{x})\left[\boldsymbol{\nabla}(\boldsymbol{B} \cdot \boldsymbol{\pi})-\frac{2}{\hbar}(\boldsymbol{E} \times \boldsymbol{\pi}) \times \boldsymbol{\pi}-i \epsilon_{j k}^{i}\left[\nabla_{i}(\boldsymbol{E} \times \boldsymbol{\pi})^{j}\right] \hat{\boldsymbol{x}}^{k}\right]\right] \\
\boldsymbol{\Lambda}_{\mathrm{CT}}^{1}= & \boldsymbol{\Lambda}_{\kappa}^{1}+\frac{\hbar}{2|\boldsymbol{\pi}|^{2}} \sqrt{|\boldsymbol{\pi}|^{2}+m^{2}}\left[\frac{2}{\hbar}(\boldsymbol{\nabla}(\boldsymbol{a} \cdot \boldsymbol{x}) \times \boldsymbol{\pi}) \times \boldsymbol{\pi}+i \epsilon_{j k}^{i}\left[\nabla_{i}(\boldsymbol{\nabla}(\boldsymbol{a} \cdot \boldsymbol{x}) \times \boldsymbol{\pi})^{j}\right] \hat{\boldsymbol{x}}^{k}\right] \\
& +\frac{\hbar}{|\boldsymbol{\pi}|^{3}}\left[\frac{2}{\hbar}\left(\boldsymbol{R}^{\prime} \times \boldsymbol{\pi}\right)+i \epsilon_{j k}^{i}\left(\nabla_{i} R^{\prime j}\right) \hat{\boldsymbol{x}}^{k}\right] \boldsymbol{\nabla} \Phi_{\mathrm{G}} \cdot \boldsymbol{\pi} \\
& -\frac{1}{|\boldsymbol{\pi}|} \boldsymbol{\nabla}\left(\boldsymbol{\nabla} \Phi_{\mathrm{G}} \cdot \boldsymbol{\pi}\right)+\frac{i \hbar}{|\boldsymbol{\pi}|^{3}} \boldsymbol{R}^{\prime} \times \boldsymbol{\nabla}\left(\boldsymbol{\nabla} \Phi_{\mathrm{G}} \cdot \boldsymbol{\pi}\right) .
\end{aligned}
$$

Given that the CT-Hamiltonian is an ultrarelativistic approximation of the original Dirac Hamiltonian, we expect that many of the terms in (4.20) and (4.21) will be small compared to the contributions due to the original Dirac Hamiltonian if we disregard those due to inhomogeneous fields. Nonetheless, there are a few noteworthy terms which should make a meaningful contribution. One of them is the fifth term in (4.20), a spin-orbit coupling due to the electric field which yields a term $\frac{1}{|\boldsymbol{\pi}|}(\kappa e / m)(\boldsymbol{E} \times \boldsymbol{\pi}) \times \boldsymbol{\pi}$. The other contributions of note are the second, fourth, and sixth terms of (4.21), which are due to the ultrarelativistic analogues of the Hehl-Ni spin-orbit coupling and gravitational energy redshift terms found in the low-energy approximation. 


\section{Chiral Transition Rate for a Relativistic Spin-1/2 Particle in a Gravi- tational Field}

It is of interest to also study the effect of inertia on the chirality precession of spin- $1 / 2$ particles, for comparison with the helicity precession. Given that the $\gamma^{5}$ operator in the chiral representation is

$$
\gamma^{5}=\left(\begin{array}{cc}
1 & 0 \\
0 & -1
\end{array}\right)
$$

the projection operators for isolating right- and left-handed states are defined as

$$
\begin{gathered}
P_{\mathrm{R}} \equiv \frac{1}{2}\left(1+\gamma^{5}\right), \quad P_{\mathrm{L}} \equiv \frac{1}{2}\left(1-\gamma^{5}\right), \\
\psi \equiv\left(\begin{array}{c}
\varphi_{\mathrm{R}} \\
\varphi_{\mathrm{L}}
\end{array}\right) .
\end{gathered}
$$

Equivalently, (5.2) can be written as

$$
P_{ \pm}=\frac{1}{2}\left(1 \pm \gamma^{5}\right)
$$

Applying the CT transformation on (5.4) leads to

$$
P_{\mathrm{CT} \pm} \approx P_{ \pm} \pm \frac{\omega(q)}{2|\boldsymbol{\pi}|} \gamma^{5} \beta(\boldsymbol{\alpha} \cdot \boldsymbol{\pi}) .
$$

Then, to leading order in $q$,

$$
\dot{P}_{\mathrm{CT} \pm} \approx \pm q\left[\frac{i}{\hbar} H_{1} \gamma^{5} \beta-\frac{1}{2}\left(1-\frac{\hbar}{|\boldsymbol{\pi}|^{2}} \boldsymbol{\sigma} \cdot \boldsymbol{R}^{\prime}\right)\left\{\frac{i}{\hbar}\left[H_{0}, h\right]+\frac{1}{|\boldsymbol{\pi}|}(\boldsymbol{\alpha} \cdot \boldsymbol{\pi}) \cdot \frac{i}{\hbar}\left[H_{0}, \beta\right] \beta \gamma^{5}\right\}\right] \beta
$$

Explicitly, the chirality transition rate (5.6) is given by (3.9) and

$$
\begin{aligned}
& \frac{1}{|\boldsymbol{\pi}|}(\boldsymbol{\alpha} \cdot \boldsymbol{\pi}) \cdot \frac{i}{\hbar}\left[H_{0}, \beta\right] \beta=\frac{2}{|\boldsymbol{\pi}|^{2}}\left[\sqrt{|\boldsymbol{\pi}|^{2}+m^{2}}[\boldsymbol{\nabla}(\boldsymbol{a} \cdot \boldsymbol{x}) \cdot \boldsymbol{\pi}+i \boldsymbol{\sigma} \cdot[\boldsymbol{\nabla}(\boldsymbol{a} \cdot \boldsymbol{x}) \times \boldsymbol{\pi}]]\right. \\
& \left.\quad-\frac{q^{3}}{\sqrt{1+q^{2}}} \frac{\hbar}{2 m}\left[[\boldsymbol{\sigma} \cdot \boldsymbol{\nabla}(\boldsymbol{a} \cdot \boldsymbol{x})] \boldsymbol{R}^{\prime} \cdot \boldsymbol{\pi}+i \boldsymbol{\nabla}(\boldsymbol{a} \cdot \boldsymbol{x}) \cdot\left(\boldsymbol{R}^{\prime} \times \boldsymbol{\pi}\right)-\boldsymbol{\sigma} \cdot\left[\boldsymbol{\nabla}(\boldsymbol{a} \cdot \boldsymbol{x}) \times\left(\boldsymbol{R}^{\prime} \times \boldsymbol{\pi}\right)\right]\right]\right] \\
& \quad+\frac{2 i}{\hbar|\boldsymbol{\pi}|^{2}}(1+\boldsymbol{a} \cdot \boldsymbol{x})\left[\sqrt{|\boldsymbol{\pi}|^{2}+m^{2}}\left(\boldsymbol{\pi} \cdot \boldsymbol{\pi}+\hbar \boldsymbol{\sigma} \cdot \boldsymbol{R}^{\prime}\right)-\frac{q^{3}}{\sqrt{1+q^{2}}} \frac{\hbar}{2 m}\left[\boldsymbol{\sigma} \cdot\left[-i \hbar \boldsymbol{\nabla}\left(\boldsymbol{R}^{\prime} \cdot \boldsymbol{\pi}\right)+\left(\boldsymbol{R}^{\prime} \cdot \boldsymbol{\pi}\right) \boldsymbol{\pi}\right]\right.\right. \\
& \left.\left.\quad+\hbar\left[\left[\nabla_{k}\left(\boldsymbol{R}^{\prime} \times \boldsymbol{\pi}\right)^{k}\right]+i \sigma^{k} \epsilon_{j k}^{i}\left[\nabla_{i}\left(\boldsymbol{R}^{\prime} \times \boldsymbol{\pi}\right)^{j}\right]\right]+\boldsymbol{\sigma} \cdot\left[\left(\boldsymbol{R}^{\prime} \times \boldsymbol{\pi}\right) \times \boldsymbol{\pi}\right]\right]\right] \\
& \quad+\frac{2}{|\boldsymbol{\pi}|}\left[\left(\boldsymbol{\nabla} \cdot \boldsymbol{\nabla} \Phi_{\mathrm{G}}\right)+i \sigma^{k} \epsilon^{i j}{ }_{k}\left(\nabla_{i} \nabla_{j} \Phi_{\mathrm{G}}\right)\right]+\frac{2 i}{\hbar|\boldsymbol{\pi}|}\left[\nabla \Phi_{\mathrm{G}} \cdot \boldsymbol{\pi}-i \boldsymbol{\sigma} \cdot\left[\nabla \Phi_{\mathrm{G}} \times \boldsymbol{\pi}\right]\right] .
\end{aligned}
$$

Excluding the contributions from the anomalous magnetic moment terms, it is clear from (5.6) that

$$
\left.\dot{P}_{\mathrm{CT} \pm}\right|_{m=0}=0,
$$

and so the chirality is a constant of the motion for massless particles. This difference between (4.13) and (5.8) strongly suggests that helicity and chirality describe entirely different physical processes, and their respective interpretations may require closer investigation. A comprehensive study of chirality transitions in a Schwarzschild field is presented in [42]. 


\section{Conclusions}

Inertial-gravitational fields affect quantum particles in different ways. They interact with particle spins and give rise to quantum phases that can be measured in principle by interferometric means. In this case $\Phi_{\mathrm{G}}$ must be calculated over a closed space-time path that can be obtained, for instance, by comparing the phase of a particle at the final position $P_{f}$ at the final time $t_{f}$ with that of an identical particle at the the same final point $P_{f}$, but at the initial time $t_{i}$.

Through the Hamiltonian, the fields can also affect the energy levels and the time evolution of observables. In the latter case, inertial fields change the helicity and chirality of particles in ideal storage rings. This has been studied in some detail in Sections 3 to 5. The results independently confirm that the spin-rotation coupling compensates the much larger contribution that comes from the $g=2$ part of the magnetic moment of a pure Dirac particle. Without this cancellation, $g-2$ experiments may be more difficult to perform with the present accuracy of $0.7 \mathrm{ppm}$ [3].

In the more general case of an inhomogeneous $\boldsymbol{\omega}$, the Mashhoon term per se essentially disappears, but a new term $1 /|\pi| \epsilon_{j k}^{i}\left(\omega_{i, 1}+i \omega_{i, 2}\right) x^{j} \pi^{k}$ contributes to the spin precession. In the lowest approximation, the spin precesses with the same angular frequency $\omega$ of the particle itself. The ratio of this new term to $\omega$ is $\simeq[(\nabla \omega) / \omega] x$. It may be possible to conceive of a physical situation in which this term can be observed, which would extend our knowledge of rotational inertia.

A second interesting result is represented by (4.13), which states that helicity is not conserved in the presence of first-order inertial and gravitational fields, even when the mass of the particle vanishes. The corresponding result (5.8) for chirality gives a vanishing result. It seems, therefore, that first-order inertia-gravitation can distinguish between helicity and chirality. This may be due to the approximation itself. It has, in fact, been mentioned that particles acquire an effective mass when immersed in gravitational fields. This result extends to inertia because, in the weak field approximation, $R=-\frac{1}{2} \partial_{\nu} \partial^{\nu} \gamma_{\mu}{ }^{\mu}$ need not vanish. In the general case, however, $R=0$ rigorously for inertial fields, but not necessarily so for true gravitational fields. Finally, it may be exceedingly difficult to subject massless fermions

to acceleration and rotation as required, for instance, by (4.14) and (4.15). In this sense, it may be said that massless fermions strive to conform to a sort of helicity conservation.

\section{Acknowledgements}

This work was supported in part by the Natural Sciences and Engineering Research Council of Canada.

\section{References}

[1] DeWitt B S 1966 Phys. Rev. Lett. 161092

[2] Papini G 1966 Nuovo Cimento 45 66; 1966 Phys. Lett. 23 418; 1967 Phys. Lett. A 24 32; 1967 Nuovo Cimento B 52136

[3] Cai Y Q and Papini G 1989 Class. Quantum Grav. 6407

[4] Hildebrandt A F, Saffren M M 1965 Proc. 9th Int. Conf. on Low-Temp. Phys. Pt. A 459; Hendricks J B and Rorschach H E Jr ibid. 466; Bol M and Fairbank W M ibid. 471; Zimmerman J E and Mercereau J E 1965 Phys. Rev. Lett. 14887

[5] Colella R, Overhauser A W and Werner S A 1975 Phys. Rev. Lett. 341472

[6] Papini G 2004 Relativity in Rotating Frames ed Guido Rizzi and Matteo Luca Ruggiero (Kluwer Academic Publishers: Dordrecht) 335 
[7] Papini G 2002 Phys. Rev. D 65077901

[8] Bell J S and Leinaas J 1987 Nucl. Phys. B284 488

[9] Cai Y Q, Lloyd and Papini G 1993 Phys. Lett. A178 225

[10] Bennett G W et al. (Muon (g-2) Collaboration) 2002 Phys. Rev. Lett. 89 101804; Brown H N et al. (Muon (g-2) Collaboration) 2001 Phys. Rev. Lett. 86 2227; 2000 Phys. Rev. D 62091101

[11] Mashhoon B 1988 Phys. Rev. Lett. 612639

[12] Mashhoon B 1989 Phys. Lett. A139 103

[13] Cai Y Q and Papini G 1991 Phys. Rev. Lett. 66 1259; 1992 Phys. Rev. Lett. 683811

[14] Papini G 1994 Proc. of the 5th Canadian Conference on General Relativity and Relativistic Astrophysics ed R B Mann and R G McLenaghan (World Scientific: Singapore) 107

[15] Mergulhão Carlos Jr 1994 General Relativity and Gravitation 27657

[16] Aldovrandi R, Matsas G E A, Novaes S F and Spehler D 1994 Phys. Rev. D 502645

[17] Singh D and Papini G 2000 Nuovo Cimento 115223

[18] De Oliveira C G and Tiomno J 1962 Nuovo Cimento 24672

[19] Peres A 1962 Suppl Nuovo Cimento 24389

[20] Hehl F W and Ni W-T 1990 Phys. Rev. D42 2045

[21] Obukhov Y N 2001 Phys. Rev. Lett. 86192

[22] Werner S A, Staudenmann, J-L and Colella R 1979 Phys. Rev. Lett. 42 1103; Page L A 1975 ibid. 35543

[23] Bonse U and Wroblewski T 1983 Phys. Rev. Lett. 511401

[24] Papini G 2002 Advances in the interplay between quantum and gravity physics ed Peter G. Bergmann and V. de Sabbata (Kluwer Academic: Dordrecht) 317; gr-qc/0110056

[25] Papini G and Lambiase G 2002 Phys. Lett. A 294175

[26] Foldy L L and Wouthuysen S A 1950 Phys. Rev. 7830

[27] Cini M and Touschek B 1958 Nuovo Cimento 7422

[28] Cai Y Q and Papini G 1989 Class. Quantum Grav. 6407

[29] Cai Y Q and Papini G 1989 Mod. Phys. Lett. A4 1143; 1990 Class. Quantum Grav. 7 269; 1990 Gen. Rel. Grav. 22 259; Papini G 1990 Quantum Mechanics in Curved Space-Time ed J Audretsch and V de Sabbata (Plenum Press: New York) 473

[30] Berry M V 1984 Proc. of the Royal Soc., London A392 45

[31] De Felice F and Clarke C J S 1990 Relativity on Curved Manifolds (Cambridge University Press: New York)

[32] Bjorken J D and Drell S D 1964 Relativistic Quantum Mechanics (McGraw-Hill: San Francisco)

[33] Bose S K, Gamba A and Sudarshan E C 1959 Phys. Rev. 1131661

[34] Itzykson C and Zuber J-B 1980 Quantum Field Theory (McGraw-Hill: Toronto) 
[35] Riley K F, Hobson, M P and Bence S J 1997 Mathematical Methods for Physics and Engineering (Cambridge University Press: New York)

[36] Mashhoon B 2003 gr-qc/0301065

[37] Irvine W M 1964 Physica 301160

[38] Sakurai J J 1967 Advanced Quantum Mechanics (Addison-Wesley: New York)

[39] Montague B W 1984 Phys. Rep. 1131

[40] Weyl H 1929 Zeits. f. Physik 56330

[41] Obukhov Y N 2002 Fortsch. Phys. 50711

[42] Singh D $2004 \quad g r-q c / 0401044$

[43] Bennett G W et al. (Muon (g-2) Collaboration) 2004 hep-ex/0401008 